\title{
Reasoning about Noisy Sensors and Effectors in the Situation Calculus*
}

\author{
Fahiem Bacchus \\ Dept. Computer Science \\ University of Waterloo \\ Waterloo, Ontario \\ Canada, N2L 3G1 \\ fbacchus@logos.uwaterloo.ca
}

\author{
Joseph Y. Halpern \\ Dept. Computer Science \\ Cornell University \\ Ithaca, NY 14850 \\ halpern@cs.cornell.edu
}

\author{
Hector J. Levesque \\ Dept. Computer Science \\ University of Toronto \\ Toronto, Ontario \\ Canada, M5S 3H5 \\ hector@cs.toronto.edu
}

\section{March 6, 2018}

\begin{abstract}
Agents interacting with an incompletely known world need to be able to reason about the effects of their actions, and to gain further information about that world they need to use sensors of some sort. Unfortunately, both the effects of actions and the information returned from sensors are subject to error. To cope with such uncertainties, the agent can maintain probabilistic beliefs about the state of the world. With probabilistic beliefs the agent will be able to quantify the likelihood of the various outcomes of its actions and is better able to utilize the information gathered from its error-prone actions and sensors. In this paper, we present a model in which we can reason about an agent's probabilistic degrees of belief and the manner in which these beliefs change as various actions are executed. We build on a general logical theory of action developed by Reiter and others, formalized in the situation calculus. We propose a simple axiomatization that captures an agent's state of belief and the manner in which these beliefs change when actions are executed. Our model displays a number of intuitively reasonable properties.
\end{abstract}

\footnotetext{
${ }^{*}$ The work of Fahiem Bacchus and Hector Levesque was supported in part by the Canadian government through their NSERC and IRIS programs. The work of Joseph Halpern supported in part by the Air Force Office of Scientific Research (AFSC), under Contracts F49620-91-C-0080 and F94620-96-1-0323 and NSF grant IRI-9625901. Much of this work was done while Halpern was at the IBM Almaden Research Center. A preliminary version of this paper appears in Proceedings of IJCAI '95, pp. 1933-1940.
} 


\section{Introduction}

An intelligent agent interacting with a dynamic and incompletely known world faces two special sorts of reasoning problems. First, because the world is dynamic, it will need to reason about change: how its actions and the actions of others affect the state of the world. For example, an agent will need to reason that if a fragile object is dropped then it will probably break, and regardless of what else happens, the object will remain broken until it is repaired. Second, because the world is incompletely known, the agent will need to make do with partial descriptions of the state of the world. As a result, the agent will often need to augment what it knows by performing perceptual actions, using sensors of some sort. Unfortunately, both the effectors that the agent uses to modify the world and the sensors it uses to sense the world will in practice be subject to uncertainty, i.e., they will be noisy. For example, a robotic agent may not know initially how far away it is from the nearest wall, but may have a sensor that it can use to obtain information about this distance. Because the sensor is noisy, a reading of, say, "3.1 meters" does not guarantee that the agent is actually 3.1 meters from the wall, although it should serve to increase the agent's degree of belief in that fact. It may need to read this sensor (or additional sensors) a number of times to get a sufficiently reliable measurement. Similarly, if it attempts to move 0.7 meters towards the wall, it may well end up moving 0.8 meters due to inaccuracies in its effectors. Nevertheless, its degree of belief that it is closer to the wall should increase. In this paper, we propose a representational formalism to capture the reasoning required to keep the agent's beliefs about the world synchronized with the effects of the various actions it performs. Without such a synchronization the agent would be unable to modify its environment in any purposeful manner.

Somewhat surprisingly, although the importance of dealing with dynamic and incompletely known worlds has long been argued within AI, very few adequate representation formalisms have emerged. We can classify existing ones into two broad camps. On the one hand, we have probabilistic formalisms such as Bayesian nets [22] for dealing with uncertainty in general, and the uncertainty that would arise from noisy sensors in particular. However, with the exceptions noted below, these probabilistic formalisms have not attempted to incorporate a general model of action, i.e., representing what does and does not change as the result of performing an action. In addition, while it is possible to express in these formalisms probabilistic dependencies among variables, which are in essence atomic propositions, it is not easy to deal with many other forms of incomplete knowledge. For example, it is difficult to say that one of two conditions holds, or that all objects of a certain type have a certain property when it is not known what those objects are. Logical formalisms, on the other hand, with features like disjunction and quantification, are well suited for expressing incomplete knowledge of this type. Moreover, logical formalisms like dynamic logics, process logics, or the situation calculus, allow us to reason about the prerequisites and effects of actions.

These observations suggest that it would be useful to have a formalism that allows us to combine actions, knowledge, (probabilistic) beliefs, and first-order reasoning. There has been work that puts some of these components together. For example, the frameworks of Bacchus [1] and Halpern [8] combines first-order reasoning and probability, but this work does not explicitly address reasoning about actions. The framework of Halpern and Tuttle [10] has 
probability, knowledge, time, and actions, but it is not a first-order formalism. Finally, the action network formalism of Darwiche and Goldszmidt [2] extends Bayesian nets to allow probabilistic reasoning about action and observation sequences and their effects, but it also is not a first-order formalism, nor can it easily deal with other forms of incomplete knowledge like disjunction.

In this paper, we propose a framework that combines first-order reasoning, with reasoning about knowledge, probability, and actions. For reasoning about actions we start with a variant of the situation calculus [20] that incorporates the solution to the frame problem proposed by Reiter [26]. Building on this and on the work of Scherl and Levesque [28], who incorporated knowledge and perceptual actions into the situation calculus, we show how probabilities can be added and how the effects of actions on an agent's probabilistic beliefs can be modeled.

We base our work on the situation calculus as it has proved to be a very convenient formalism for modeling actions, their prerequisites, and their effects, and for modeling incomplete knowledge of the state of the world. Of course, our approach inherits these features. In addition, it allows us to build on previous work done in this framework and to take advantage of parallel developments. For example, although Reiter's solution to the frame problem is limited in a number of ways, it has been extended to handle aspects of the ramification problem [17], agent ability [13], continuous time [25], and perceptual actions [28]. Another extension of the theory to deal with complex actions (sequence, iterations, recursive procedures, non-determinism, etc.), briefly described in Section 3, has led to a novel logic programming language called GOLOG. GOLOG has proved to be useful for describing high-level robot and softbot control [15]. An implementation of GOLOG exists and a number of programs (including a banking agent softbot application [14]) currently run in simulation mode. A mail delivery application written in GOLOG also runs on two different robot platforms 31. We expect that the extensions we make here can be incorporated into this framework as well.

Independently of the situation calculus, however, our formalism demonstrates an interesting interaction between actions and differing mechanisms for updating probabilistic beliefs. And as we hope to make clear from our presentation, much of what we do here could be carried out in other logical frameworks.

The rest of the paper is organized as follows. In the next section, we briefly review the theory of action in terms of which our account is formulated: the situation calculus and the solution to the frame problem proposed by Reiter. In Section 3, we examine how non-

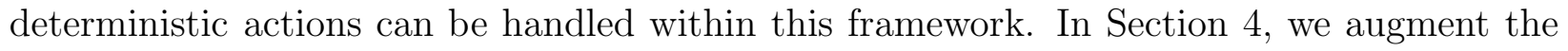
framework by adding the notion of the agent's epistemic state. In Section 5 we show how probabilities can be added, and present a simple formalization within the situation calculus of the degree of belief an agent has in propositions expressed as logical formulas. This allows us to formalize in more quantitative terms the changes in belief that arise when dealing with noisy sensors and effectors. Examples of the formalism at work are presented in Section 7 , and some conclusions are drawn in Section 8 . 


\section{A Theory of Action}

Our account of sensors is formulated as a logical theory in an extended version of the situation calculus. The situation calculus [20] is a many-sorted dialect of the predicate calculus (with some second-order features), containing sorts for (among other things) situations, which are like the possible worlds of modal logic, for primitive deterministic actions, and, since we will be dealing with probabilities, for real numbers.

The situation calculus is specifically designed for representing dynamically changing worlds. All changes to the world are the result of named actions. A possible world history, which is simply a sequence of actions, is represented by a first-order term called a situation. The constant $S_{0}$ is used to denote the initial situation, namely that situation in which no actions have yet occurred. There is a distinguished binary function symbol $d o$; $d o(\alpha, s)$ denotes the successor situation to $s$ resulting from performing the action $\alpha$. Actions may be parameterized. For example, put $(x, y)$ might stand for the action of putting object $x$ on object $y$, in which case $d o(\operatorname{put}(A, B), s)$ denotes that situation resulting from placing $A$ on $B$ when in situation $s$. Notice that in the situation calculus, actions are denoted by first order terms, and situations (world histories) are also first order terms. For example, $d o\left(\operatorname{putdown}(A), d o\left(\operatorname{walk}(L), d o\left(\operatorname{pickup}(A), S_{0}\right)\right)\right)$ is a situation denoting the world history consisting of the sequence of actions $[\operatorname{pickup}(A)$, walk $(L)$, putdown $(A)]$.

Relations whose truth values vary from situation to situation, called relational fluents, are denoted by predicate symbols taking a situation term as their last argument. For example, IsCarrying (robot, $p, s)$, meaning that the robot is carrying package $p$ in situation $s$, is a relational fluent. Functions whose denotations vary from situation to situation are called functional fluents. They are denoted by function symbols with an extra argument taking a situation term, as in position (robot, $s$ ), i.e., the robot's location in situation $s$. Finally, we use the distinguished predicate $\operatorname{Poss}(a, s)$ to state that action $a$ is possible to execute in situation $s$.

\subsection{Actions}

Actions have preconditions - necessary and sufficient conditions that characterize when the action is physically possible. For example, in a blocks world, we might have:円

$$
\begin{aligned}
& \operatorname{Poss}(\operatorname{pickup}(x), s) \\
& \quad \equiv \neg \exists z . \operatorname{Holding}(z, s) \wedge \operatorname{NextTo}(x, s) \wedge \neg \operatorname{Heavy}(x),
\end{aligned}
$$

which says that an object $x$ can be picked up if and only if the agent is not currently holding something, it is next to $x$, and $x$ is not heavy.

Definition 1: [Precondition Axioms] Precondition axioms are axioms of the form

$$
\operatorname{Poss}(a, s) \equiv \phi(a, s)
$$

Here $\phi$ is a formula that specifies the necessary and sufficient properties of the situation $s$ and the action $a$, under which $a$ can be executed in $s$.

\footnotetext{
${ }^{1}$ In formulas, free variables are considered to be universally quantified. This convention will be followed throughout the paper.
} 
The manner in which the actions modify the world are specified by their effect axioms. These describe the effects of a given action on the fluents. For example, a robot dropping a fragile object causes it to be broken:

$$
\operatorname{Poss}(\operatorname{drop}(r, x), s) \wedge \operatorname{Fragile}(x, s) \supset \operatorname{Broken}(x, \operatorname{do}(\operatorname{drop}(r, x), s)) .
$$

Exploding a bomb next to an object causes it to be broken:

$$
\operatorname{Poss}(\operatorname{explode}(b), s) \wedge \operatorname{Next} T o(b, x, s) \supset \operatorname{Broken}(x, \operatorname{do}(\operatorname{explode}(b), s)) \text {. }
$$

A robot repairing an object causes it to be not broken:

$$
\operatorname{Poss}(\operatorname{repair}(r, x), s) \supset \neg \operatorname{Broken}(x, d o(\operatorname{repair}(r, x), s)) .
$$

\subsection{The Frame Problem}

As first observed by McCarthy and Hayes [20], axiomatizing a dynamic world requires more than just action precondition and effect axioms. So-called frame axioms are also necessary. These specify the action invariants of the domain, namely, those fluents that remain unaffected by a given action. For example, an object's colour is not affected by a robot dropping something:

$$
\operatorname{Poss}(\operatorname{drop}(r, x), s) \wedge \operatorname{Colour}(y, s)=c \supset \operatorname{Colour}(y, \operatorname{do}(\operatorname{drop}(r, x), s))=c .
$$

A frame axiom describing how the fluent Broken is unaffected:

$$
\begin{aligned}
& \operatorname{Poss}(\operatorname{drop}(r, x), s) \wedge \neg \operatorname{Broken}(y, s) \wedge y \neq x \vee \neg \operatorname{Fragile}(y, s) \\
& \supset \neg \operatorname{Broken}(y, \operatorname{do}(\operatorname{drop}(r, x), s)) .
\end{aligned}
$$

The problem introduced by the need for such frame axioms is that we can expect a great many of them. Relatively few actions will affect the truth value of a given fluent; all other actions leave the fluent unchanged. For example, an object's colour is not changed by picking things up, opening a door, going for a walk, electing a new prime minister of Canada, etc. This is problematic for the axiomatizer who must think of all these axioms; it is also problematic for any automated reasoning process as it must reason efficiently in the presence of so many frame axioms.

Suppose that the person responsible for axiomatizing an application domain has specified all of the causal laws for the world being axiomatized. More precisely, suppose they have succeeded in writing down all the effect axioms, i.e., for each fluent $F$ and each action $A$ that can cause $F$ 's truth value to change, they have written axioms of the form

$$
\operatorname{Poss}(A, s) \wedge R(\vec{x}, s) \supset(\neg) F(\vec{x}, d o(A, s)),
$$

where $R$ is a first-order formula specifying the contextual conditions under which the action $A$ will have its specified effect on $F$.

A reasonable solution to the frame problem would be a systematic procedure for generating, from these effect axioms, all the frame axioms. If possible, we would also want a parsimonious representation for these frame axioms (because in their simplest form, there are too many of them). 


\subsection{A Simple Solution to the Frame Problem}

By appealing to earlier ideas of Haas [7], Schubert [29], and Pednault [24], Reiter [26] proposed a simple solution to the frame problem, which we employ in this work. It is best illustrated with an example. Suppose that Equations 1, 2, and 3 are all the effect axioms for the fluent Broken, i.e., they describe all the ways that any action can change the truth value of Broken. We can rewrite (1) and (2) in the logically equivalent form:

$$
\begin{gathered}
\operatorname{POss}(a, s) \wedge \exists r . a=\operatorname{drop}(r, x) \wedge \operatorname{Fragile}(x, s) \\
\vee \exists b . a=\operatorname{explode}(b) \wedge \operatorname{NextTo}(b, x, s) \\
\supset \operatorname{Broken}(x, \operatorname{do}(a, s)) .
\end{gathered}
$$

Similarly, consider the negative effect axiom for Broken, (3); this can be rewritten as:

$$
\operatorname{Poss}(a, s) \wedge \exists r . a=\operatorname{repair}(r, x) \supset \neg \operatorname{Broken}(x, d o(a, s)) .
$$

In general, we can assume that the effect axioms for a fluent $F$ have been written in the forms:

$$
\begin{gathered}
\operatorname{Poss}(a, s) \wedge \gamma_{F}^{+}(\vec{x}, a, s) \supset F(\vec{x}, d o(a, s)), \\
\operatorname{Poss}(a, s) \wedge \gamma_{F}^{-}(\vec{x}, a, s) \supset \neg F(\vec{x}, d o(a, s)) .
\end{gathered}
$$

Here $\gamma_{F}^{+}(\vec{x}, a, s)$ is a formula describing the conditions under which doing the action $a$ in situation $s$ causes the fluent $F$ to become true in the successor situation $d o(a, s)$; similarly $\gamma_{F}^{-}(\vec{x}, a, s)$ describes the conditions under which performing $a$ in $s$ causes $F$ to become false in the successor situation. Reiter's solution to the frame problem rests on a completeness assumption, which is that the causal axioms (4) and (5) characterize all the conditions under which action $a$ can lead to a fluent $F(\vec{x})$ becoming true (respectively, false) in the successor situation. In other words, axioms (4⿴) and (5) describe all the causal laws affecting the truth values of the fluent $F$. Therefore, if action $a$ is possible and $F(\vec{x})$ 's truth value changes from false to true as a result of doing $a$, then $\gamma_{F}^{+}(\vec{x}, a, s)$ must be true and similarly for a change from true to false. Reiter [26] shows how to automatically derive a successor-state axiom from the causal axioms (4) and (5) and the completeness assumption.

Definition 2: [Successor-State Axioms] Successor-state axioms are axioms of the following form

$$
\begin{aligned}
& \operatorname{Poss}(a, s) \supset F(\vec{x}, d o(a, s)) \\
& \quad \equiv \gamma_{F}^{+}(\vec{x}, a, s) \vee\left(F(\vec{x}, s) \wedge \neg \gamma_{F}^{-}(\vec{x}, a, s)\right) .
\end{aligned}
$$

for relational fluents $F$, and of the form

$$
\begin{aligned}
& \operatorname{POss}(a, s) \supset f(\vec{x}, d o(a, s))=z \\
& \quad \equiv \gamma_{f}(z, \vec{x}, a, s)
\end{aligned}
$$

for functional fluents $f$. These axioms characterize the state of a fluent $F$ (or the value of a functional fluent $f$ ) in the successor situation given properties of the current state and the action executed. 
The successor-state axiom for $F$ is a solution to the frame problem for that fluent. Notice that this axiom universally quantifies over actions $a$. In fact, the ability to universally quantify over actions is one of the keys to obtaining a parsimonious solution to the frame problem.

Applying this to our example about breaking things, we obtain the following successorstate axiom:

$$
\begin{aligned}
& \operatorname{Poss}(a, s) \supset \operatorname{Broken}(x, d o(a, s)) \\
& \equiv \exists r . a=\operatorname{drop}(r, x) \wedge \operatorname{Fragile}(x, s) \\
& \vee \exists b . a=\operatorname{explode}(b) \wedge \operatorname{NextTo}(b, x, s) \\
& \vee \operatorname{Broken}(x, s) \wedge \neg \exists r . a=\operatorname{repair}(r, x) .
\end{aligned}
$$

It is important to note that the above solution to the frame problem presupposes that there are no state constraints, as for example the blocks world constraint: $\forall s$. On $(x, y, s) \supset$ $\neg O n(y, x, s)$. Such constraints can implicitly contain effect axioms (so-called indirect effects), in which case the above completeness assumption will not be true. The assumption that there are no state constraints in the axiomatization of the domain will be made throughout this paper.

In general, for any application, we use what is called in [17] a basic action theory consisting of the following axioms:

- axioms describing the initial situation - what is true initially, before any actions have occurred. This is any finite set of sentences that mention only the situation term $S_{0}$.

- action-precondition axioms (Defn. 1), one for each primitive action

- successor-state axioms (Defn. 凤), one for each fluent

- unique-name axioms for the primitive actions (saying that primitive actions with distinct names are different)

- a set of domain-independent foundational axioms for the situation calculus [17]. These are used to state that the situations are all and only those reachable from $S_{0}$ by performing a finite sequence of actions.

\section{Nondeterministic Actions}

In the description of the situation calculus in the previous section, actions are taken to be deterministic in the sense that it is assumed that there exists a unique successor state for any action executed. To model noisy sensors and effectors, we first show how nondeterministic actions can be handled within this framework without giving up the simple solution to the frame problem outlined above.

Nondeterministic actions are treated as being the actual execution of one of a range of primitive (deterministic) actions. Nondeterminism arises from the fact that we do not know exactly which primitive action was actually executed. Thus, when a nondeterministic

\footnotetext{
${ }^{2}$ In [17], the approach discussed in this section is extended to deal with some forms of state constraints, by compiling their effects into the successor-state axioms.
} 
action is executed by the agent, the current situation will have a set of successor states, one successor state for each possible primitive action. Nevertheless, we retain the property that each of the underlying primitive actions yields a unique successor state. Hence, the solution to the frame problem above can be applied to each of these states individually, and any properties than can be shown to hold of every successor state will then be provable consequences of the nondeterministic action.

The idea can be illustrated by imagining a simple robot that can move along an unbounded one-dimensional surface. In the deterministic case, we might have a functional fluent position $(s)$ and an action exact-advance $(x)$ that changes the position of the robot by some amount $x$. We could then write the following successor-state axiom for position:

$$
\begin{gathered}
\operatorname{Poss}(a, s) \supset \operatorname{position}(d o(a, s))= \\
\text { if } \exists x . a=\operatorname{exact-advance}(x) \\
\text { then position }(s)+x \\
\text { else position }(s),
\end{gathered}
$$

assuming that exact-advance is the only action affecting position. [

The action exact-advance is deterministic - executing it yields a unique successor state. Suppose instead we want to model a nondeterministic action noisy-advance $(x)$, which results in the agent moving a distance $y$ which is approximately equal to $x$. Since we only allow deterministic primitive actions in our framework, we view noisy-advance $(x)$ as the union of all the possible moves that the agent could actually make, given that it tries to move $x$. To capture this, we assume that the language contains primitive actions of the form advance $(x, y)$, where $x$ is the nominal distance the agent is trying to move, by sending an appropriate command to its on-board effectors, while $y$ is the actual distance moved. The agent has control over the nominal distance, but no direct control over the actual distance moved. So, in fact, the agent cannot execute an instance of advance $(x, y)$ directly.

Nevertheless, we can write a successor-state axiom for position assuming that it can be changed only by the action advance. The axiom is the same as above except that the position is changed by the actual distance moved and the nominal distance is ignored.

$$
\begin{gathered}
\operatorname{Poss}(a, s) \supset \operatorname{position}(d o(a, s))= \\
\text { if } \exists x, y \cdot a=\operatorname{advance}(x, y) \\
\text { then position }(s)+y \\
\text { else position }(s) .
\end{gathered}
$$

Although the agent cannot execute an instance of advance directly, it can activate its effectors specifying a particular nominal value for the distance to be moved. This corresponds

\footnotetext{
${ }^{3}$ We are taking some liberties here with notation and the scope of variables; the if-then-else formula should be viewed as an abbreviation for the formula

$$
\begin{aligned}
\operatorname{Poss}(a, s) & \supset \operatorname{position}(d o(a, s))=z \\
\equiv \exists x . & =\text { exact-advance }(x) \wedge z=\operatorname{position}(s)+x \\
\vee & \neg x . a=\operatorname{exact-advance}(x) \wedge z=\operatorname{position}(s) .
\end{aligned}
$$
}


to executing a nondeterministic action: execute advance $(x, y)$ for a fixed value of $x$ and a nondeterministic choice of $y$.

To specify the execution of such actions we need to expand our notation for do to allow for more than one potential successor state. The first step is to allow more complex actions. Starting with primitive actions (the ones which already have names in the language), we form more complex actions by closing off under sequencing and nondeterministic choice). In particular, if $\delta_{1}$ and $\delta_{2}$ are actions, then so are $\delta_{1} ; \delta_{2}$ (intuitively, perform $\delta_{1}$ and then $\delta_{2}$ ), $\delta_{1} \mid \delta_{2}$ (intuitively, nondeterministically choose one of $\delta_{1}$ and $\delta_{2}$ and perform it), and $\pi x . \delta_{1}$ (intuitively, perform $\delta_{1}$ for some nondeterministically chosen value of $x$; this is particularly interesting if $x$ is a free variable in $\delta$ ). Given a (complex) action $\delta$, we follow the approach taken in GOLOG [15] and define $D o\left(\delta, s, s^{+}\right)$as an abbreviation for a situation calculus formula which intuitively reads " $s^{+}$is a possible final situation arising from the execution of action $\delta$ in situation $s "$. Do is defined by the following recursive expansions:

1. If $\delta$ is a primitive action like exact-advance $(x)$ or advance $(x, y)$, then the expansion of $D o\left(\delta, s, s^{+}\right)$is:

$$
D o\left(a, s, s^{+}\right) \stackrel{\text { def }}{=} \operatorname{Poss}(a, s) \wedge s^{+}=d o(a, s) .
$$

2. If $\delta$ is of the form $\delta_{1} ; \delta_{2}$, then the expansion is

$$
D o\left(\left[\delta_{1} ; \delta_{2}\right], s, s^{+}\right) \stackrel{\text { def }}{=} \exists s^{\prime} . D o\left(\delta_{1}, s, s^{\prime}\right) \wedge D o\left(\delta_{2}, s^{\prime}, s^{+}\right) .
$$

3. If $\delta$ is of the form $\delta_{1} \mid \delta_{2}$, then the expansion is

$$
D o\left(\delta_{1} \mid \delta_{2}, s, s^{+}\right) \stackrel{\text { def }}{=} D o\left(\delta_{1}, s, s^{+}\right) \vee D o\left(\delta_{2}, s, s^{+}\right) .
$$

4. If $\delta$ is of the form $\pi x \cdot \delta^{\prime}$, then the expansion is

$$
D o\left(\left[\pi x . \delta^{\prime}\right], s, s^{+}\right) \stackrel{\text { def }}{=} \exists x . D o\left(\delta^{\prime}, s, s^{+}\right) .
$$

This expansion allows for a different successor state for each value of $x$.

Now we can formalize a nondeterministic move action noisy-advance $(x)$ that is directly executable by the agent:

$$
\operatorname{noisy-advance}(x) \stackrel{\text { def }}{=} \pi y \text {. advance }(x, y) \text {. }
$$

In this form, noisy-advance does not give the agent any control over its movements since we have not specified any relationship between the nominal value $x$ asked for and the actual value $y$ achieved. One reasonable constraint between these two values might be to assert that there is an absolute bound on the effector's error. That is, there is a bound on the difference between the value asked for and the value achieved. This can be captured by asserting the following precondition axiom for advance:

$$
\operatorname{Poss}(\operatorname{advance}(x, y), s) \equiv|x-y| \leq b,
$$

\footnotetext{
${ }^{4}$ Remember that $d o(\ldots)$ is defined to be a first-order term. Hence, it can denote only a single individual, in this case a single situation.
} 
where $b$ is the magnitude of the maximum possible error. This axiom says that it is impossible to execute advance actions in which the actual value moved is more that $b$ units different from the value asked for. When this precondition is combined with the definition of the nondeterministic action noisy-advance, it limits the choice of advance actions that can arise. Combining this precondition axiom, the definition of noisy-advance, and the expansions for $D o$, it is not difficult to see that the action noisy-advance $(x)$ can never yield a successor state in which the agent has moved an amount outside the range $[x-b, x+b]$. More formally we have as a direct entailment that

$$
D o\left(\operatorname{noisy-advance}(x), s, s^{+}\right) \supset\left|\operatorname{position}\left(s^{+}\right)-\operatorname{position}(s)-x\right| \leq b .
$$

In Section 5, we demonstrate how a probabilistic relationship can be specified between the nominal and actual values so as to capture the fact that these two values are correlatedalthough the agent's actions do not always have exactly their intended effect they do have an effect that is correlated with what was intended.

Finally, it should be noted that we have added nothing to the previous situation calculus story except a few convenient abbreviations. Since the execution of any complex action ultimately reduces to the execution of some collection of deterministic actions, we can apply the previous solution to the frame problem to each of the possible final situations.

\section{Adding Knowledge}

When the agent executes a nondeterministic action like the noisy-advance action described above, the particular deterministic action that ends up being executed remains unknown to the agent. Hence, it is clear that in the presence of such actions (and in the presence of perceptual actions) there needs to be a distinction between the state of the world and the information the agent has about the state of the world. We would like to be able to talk about the effect of actions like advance on what an agent or robot knows or believes about where it is located. For example, doing a noisy-advance decreases the robot's certainty about its position, but doing a sensing action increases it (in a manner to be described below). To accomplish this, we need to be able to characterize and reason about the agent's knowledge and beliefs about the world, i.e., the agent's epistemic state, and to distinguish this from the actual state of the world.

To talk about the actual state of the world in the situation calculus is easy: the current situation captures the current state of the world. To capture the agent's epistemic state we employ some standard ideas from modal logics of knowledge and belief. (See [6] for an introduction, and section 7.2 for a discussion of how the approach taken here relates to the model of knowledge presented there.) In modal logic, an agent has a binary possibility relation or accessibility relation on possible worlds. The agent is then said to know a fact $\phi$ in world (or situation) $s$ if $\phi$ is true at all the worlds the agent considers possible, as captured by the possibility relation. It is well known that various properties of knowledge can be captured by placing constraints on the possibility relation. In particular, if we take the possibility relation to be transitive and Euclidean, the agent has positive and negative introspection, so it knows what it knows and knows what it does not know. 
These ideas were first applied in the context of the situation calculus by Moore [21, who introduced a binary fluent $K$ into the language to capture the possibility relation of modal logic. That is, $K\left(s^{\prime}, s\right)$ holds if, in situation $s$, the agent considers situation $s^{\prime}$ to be possible. As in modal logic, we say that the agent knows $\phi$ in situation $s$ if $\phi\left(s^{\prime}\right)$ holds in all situations $s^{\prime}$ that the agent considers possible in $s$ (i.e., such that $K\left(s^{\prime}, s\right)$ holds). Moore's approach was extended and integrated with Reiter's solution to the frame problem by Scherl and Levesque [28]. The key issue is defining an appropriate successor-state axiom for $K$, that specifies how the fluent changes after doing an action $a$ in some situation $s$. In other words, we need to specify what has to be true of $s^{\prime+}$ for $K\left(s^{\prime+}, d o(a, s)\right)$ to hold.

Scherl and Levesque do this by dividing actions into ordinary ones (like moving) and knowledge-producing ones intended to change only the $K$ fluent. However, their characterization entails knowledge of the action executed; i.e., the agent always comes to know what action (of either type) was executed. We need to model the case where nondeterministic actions are being executed by the agent, and the agent does not come to know the exact action being executed. For example, if the action happens to be advance $(x, y)$, we do not expect the agent to know that this action was performed, since that would mean knowing the actual distance $y$ moved. Rather, we expect the agent to know only the nominal value $x$ that it tried to move and whatever constraints this places on $y$. That is, as a result of performing this action, the agent learns just that it tried to move $x$.

Another way of thinking about this is that, after doing advance $(x, y)$, the agent knows only that advance $\left(x, y^{\prime}\right)$ was performed for some compatible value $y^{\prime}$. To formalize this idea, we use a special predicate OI $\left(a, a^{\prime}, s\right)$, meaning $a$ and $a^{\prime}$ are observationally indistinguishable, i.e., cannot be distinguished by the agent. We assume that as part of the background theory, the user specifies a collection of observation-indistinguishability axioms characterizing this predicate.

Definition 3 : [Observation-Indistinguishability Axioms] Observation-indistinguishability axioms are axioms of the form

$$
\text { OI }\left(a, a^{\prime}, s\right) \equiv \phi\left(a, a^{\prime}, s\right)
$$

one for each action $a$. Here $\phi$ is a formula that characterizes the relationship between actions $a$ and $a^{\prime}$ that makes them indistinguishable in situation $s$. These axioms specify the set of actions $a^{\prime}$ that the agent has no ability to discriminate from action $a$ in situation $s$. That is, in situation $s$ the agent cannot tell if action $a$ or $a^{\prime}$ was executed.

For ordinary actions, i.e., primitive actions for which the agent knows when they are performed and knows all of their consequences, this axiom would say that Oi holds iff $a=a^{\prime}$. For example, if $\operatorname{pickup}(x)$ is an ordinary action, we would have

$$
\text { OI }\left(\operatorname{pickup}(x), a^{\prime}, s\right) \equiv a^{\prime}=\operatorname{pickup}(x) \text {. }
$$

For actions like advance $(x, y)$, however, the axiom would be

$$
\text { OI }\left(\operatorname{advance}(x, y), a^{\prime}, s\right) \equiv \exists y^{\prime} \cdot a^{\prime}=\operatorname{advance}\left(x, y^{\prime}\right)
$$


This says that, for example, the agent cannot distinguish between the executions of the actions advance $(3.0,3.2)$ and advance $(3.0,2.8)$, since both involve attempting to move the same 3.0 units.

Using Or, we can specify a new successor-state axiom for $K$ that correctly handles actions like advance $(x, y)$.

Definition 4: [K's Successor-State Axiom $]$

$$
\begin{aligned}
& \operatorname{Poss}(a, s) \supset K\left(s^{+}, d o(a, s)\right) \\
& \quad \equiv \exists a^{\prime}, s^{\prime} . s^{\prime+}=d o\left(a^{\prime}, s^{\prime}\right) \wedge \operatorname{Poss}\left(a^{\prime}, s^{\prime}\right) \wedge \operatorname{OI}\left(a, a^{\prime}, s\right) \wedge K\left(s^{\prime}, s\right)
\end{aligned}
$$

This axiom says that when the agent is in situation $d o(a, s)$ (after having executed action $a$ in situation $s$ ), the set of $K$-related situations (that define its state of knowledge) are precisely those situations that are the result of executing an observationally indistinguishable action $a^{\prime}$ from some situation $s^{\prime}$ that satisfies the preconditions of $a^{\prime}$ and was $K$-related to the previous situation $s$. Intuitively, when the agent is in $s$, as far as it knows it could be in any situation $s^{\prime}$ that is $K$-related to $s$. Hence, as far as it knows it could have executed $a$ in any of these situations, and all of the $a$ successors of these situations must be in its new knowledge set. Moreover, it does not even know if it in fact executed $a$; it could have executed any $a^{\prime}$ indistinguishable from $a$, so the $a^{\prime}$ successors of these situations must also be in its new knowledge set. However, it does know that an action indistinguishable from $a$ was successfully executed. Hence, it can eliminate from its knowledge set the successors of those situations that fail to satisfy the preconditions of some $a^{\prime}$. It is not hard to show that for ordinary actions like pickup $(x)$, which are observationally indistinguishable only from themselves, this axiom reduces to the axiom given by Scherl and Levesque [28]. . ]

As we said earlier, we define knowledge to be truth in all worlds the agent considers possible. For example, to say that the agent knows in situation $s$ that object $x$ is not broken, we would state $\forall s^{\prime} . K\left(s^{\prime}, s\right) \supset \neg \operatorname{Broken}\left(x, s^{\prime}\right)$. It is convenient to introduce special syntactic machinery to express this condition:

Definition 5: $[\mathrm{KNOW}]$ Let $s_{\text {now }}$ be a special situation term. We write $\operatorname{KnOW}\left(\phi\left[s_{\text {now }}\right], s\right)$ to indicate that the agent knows $\phi\left[s_{\text {now }}\right]$ in situation $s$. Thus, $\operatorname{KNOW}\left(\phi\left[s_{\text {now }}\right], s\right)$ is an abbreviation for the formula $\forall s^{\prime} . K\left(s^{\prime}, s\right) \supset \phi\left[s_{\text {now }} / s^{\prime}\right]$, where $s^{\prime}$ is some variable not appearing anywhere in $\phi$, and $\phi\left[s_{\text {now }} / s^{\prime}\right]$ is the result of replacing all free occurrences of $s_{\text {now }}$ in $\phi$ by $s^{\prime}$. I

For example, $\operatorname{KNOW}\left(\neg \operatorname{Broken}\left(x, s_{\text {now }}\right), s\right)$ is an abbreviation for the above formula. Notationally, it is often convenient to suppress the $s_{\text {now }}$ term and simply write $\operatorname{KNOW}(\neg \operatorname{Broken}(x), s)$, assuming that it will be clear from context where the implicit situation variable $s^{\prime}$ needs to be inserted.

\footnotetext{
${ }^{5}$ See Section 7.1 for how this axiom also correctly handles Scherl and Levesque's knowledge producing actions.

${ }^{6} \mathrm{An}$ occurrence of $s_{\text {now }}$ is considered to be bound if it appears within the scope of KNOw and free otherwise.
} 
Example 4.1: Given the axioms above, it is easy to show that after doing a noisy-advance $(x)$, the agent will know that its current position is its previous position plus $x$, give or take $b$ units. More precisely, let us assume that we have a special function prev $(s)$ which for situations other than $S_{0}$ denotes the situation immediately before $s$.] Then these formulas entail

$$
\begin{aligned}
& \operatorname{Do}\left(\text { noisy-advance }(x), s, s^{+}\right) \\
& \quad \supset \operatorname{KNOW}\left(\operatorname{position}\left(s_{\text {now }}\right) \in\left[\operatorname{position}\left(\operatorname{prev}\left(s_{\text {now }}\right)\right)+x-b, \operatorname{position}\left(\operatorname{prev}\left(s_{\text {now }}\right)\right)+x+b\right], s^{+}\right)
\end{aligned}
$$

or, in more detail,

$$
\begin{aligned}
& D o\left(\text { noisy-advance }(x), s, s^{+}\right) \\
& \supset \forall s^{+} . K\left(s^{\prime+}, s^{+}\right) \supset \exists a^{\prime}, s^{\prime} . s^{+}=\operatorname{do}\left(a^{\prime}, s^{\prime}\right) \\
& \wedge\left(\operatorname{position}\left(s^{\prime}\right)+x-b\right) \leq \operatorname{position}\left(s^{+}\right) \leq\left(\operatorname{position}\left(s^{\prime}\right)+x+b\right) .
\end{aligned}
$$

Briefly, any situation $s^{+}$that is the result of executing noisy-advance $(x)$ must have been the result of executing advance $(x, y)$ for some $y$. According to $(\mathbf{9})$, advance $(x, y)$ is executable only if $|x-y| \leq b$. Hence, from (77) we know that in $s^{+}$, the agent's position is within $b$ of its position at $s$ plus $x$. By Defn. 1 and (10), this holds in all of the situations that are $K$-related to $s^{+}$as well: all of these situations are also the result of executing advance $(x, y)$ for some $y$ with $|x-y| \leq b$. Hence, the agent knows this in $s^{+}$.

It is not hard to show that the agent would also know that this property holds before executing the noisy-advance $(x)$ action, that is, that the formula $\operatorname{KNOW}(\psi, s)$ is entailed, where $\psi$ is

$$
\begin{aligned}
& \forall s^{+} . D o\left(\text { noisy-advance }(x), s_{\text {now }}, s^{+}\right) \\
& \quad \supset \operatorname{position}\left(s^{+}\right) \in\left[\operatorname{position}\left(s_{\text {now }}\right)+x-b, \operatorname{position}\left(s_{\text {now }}\right)+x+b\right] .
\end{aligned}
$$

Observe that a noisy advance decreases positional certainty, in that even if the agent knows its current position precisely, after doing a noisy advance it will know its position only to within $b$ units. Similarly, if the agent knows its current position to within $a$ units it will know its position only to within $b+a$ units after doing a noisy advance. In particular, multiple advances add to the agent's uncertainty, so the following formula is also entailed.

$$
\begin{aligned}
& \operatorname{Do}\left(\text { noisy-advance }(x) ; \operatorname{noisy} \text {-advance }(y), s, s^{+}\right) \\
& \supset \operatorname{KNOw}\left(\operatorname { p o s i t i o n } ( s ^ { + } ) \in \left[\operatorname{position}\left(\operatorname{prev}\left(\operatorname{prev}\left(s_{\text {now }}\right)\right)\right)+x+y-2 b,\right.\right. \\
& \left.\left.\operatorname{position}\left(\operatorname{prev}\left(\operatorname{prev}\left(s_{\text {now }}\right)\right)\right)+x+y+2 b\right], s^{+}\right)
\end{aligned}
$$

\subsection{Sensing Actions}

Sensing actions can also be handled within the account we have developed so far. Sensing actions are actions that are executed primarily for the change they produce on the $K$ fluent; pure sensing actions would affect no other fluent, and would appear only in the successorstate axiom of the $K$ fluent.

\footnotetext{
${ }^{7}$ We can characterize this function by the axiom $\operatorname{prev}(d o(a, s))=s$.
} 
For example, imagine that the robot has a sensor that senses its current location in our 1dimensional world. The sensor is subject to error, so the value it returns is unlikely to be the exact location. We can model the act of using such a sensor with an action sense-position $(x, y)$ analogous to advance $(x, y)$. In this case, the nominal value $x$ would be the value appearing on the sensor when the sensing action is executed, and the actual value $y$ would be the true position. As with advance, we assume that the agent cannot observe the actual value. This gives the following observation-indistinguishability axiom for sense-position:

$$
\text { OI(sense-position } \left.(x, y), a^{\prime}, s\right) \equiv \exists y^{\prime} \cdot a^{\prime}=\operatorname{sense-position}\left(x, y^{\prime}\right) \text {. }
$$

What makes sense-position $(x, y)$ carry information is the fact that the true position $y$ places constraints on the value of $x$ that can be read from the sensor. This is captured by the precondition axiom for this action, which is

$$
\operatorname{Poss}(\text { sense-position }(x, y), s) \equiv y=\operatorname{position}(s) \wedge|x-y| \leq c .
$$

In this case, we have simply asserted that there is a bound on the error in the value read from the sensor: it must be within $c$ units of the true value.

The main difference between sense-position and advance is that with sense-position the robot does not have the ability to select either the nominal value read $x$ or the actual value $y$. The first of these is chosen nondeterministically, and depends on the error in that activation of the sensor; the second (by virtue of the above precondition axiom) is determined by the actual position of the robot. Thus, we assume that the robot merely gets to execute the nondeterministic action noisy-sense-position which takes no arguments:

$$
\text { noisy-sense-position } \stackrel{\text { def }}{=} \pi x, y \text {. sense-position }(x, y) \text {. }
$$

Example 4.2: Given the axioms above, we can show that after doing a noisy-sense-position, the agent will know what its current position is to within $c$ units. More precisely,

$$
\begin{aligned}
& D o\left(\text { noisy-sense-position, } s, s^{+}\right) \\
& \quad \supset \exists x \text { KNOw }\left(\text { position } \in[x-c, x+c], s^{+}\right),
\end{aligned}
$$

or, in more detail,

$$
\begin{aligned}
& \exists x \exists y s^{+}=d o(\text { sense-position }(x, y), s) \\
& \quad \supset \exists x \forall s^{\prime+} . K\left(s^{\prime+}, s^{+}\right) \supset(x-c) \leq \operatorname{position}\left(s^{\prime+}\right) \leq(x+c) .
\end{aligned}
$$

is entailed by these axioms.

Once again, any situation $s^{+}$that can arise from executing noisy-sense-position must be the result of executing a particular instance of sense-position $(x, y)$. According to (12), these instances are executable only if $y=\operatorname{position}(s)$ and $x \in[\operatorname{position}(s)-c, \operatorname{position}(s)+c]$. Hence, there is a fixed value $x$ (the value that will be read from the sensor) such that position $(s) \in[x-c, x+c]$. Furthermore, by (7) we see that sense-position actions do not affect the current position, so position $\left(s^{+}\right)=\operatorname{position}(s)$ and the bound holds in $s^{+}$as well. 
Now if we examine all situations $s^{+} K$-related to $s^{+}$we see that (1) they must be the result of executing an observationally indistinguishable action sense-position $\left(x^{\prime}, y^{\prime}\right)$ in a situation $s^{\prime}$ that is $K$-related to $s$, which by (11) means $x^{\prime}=x$, and (2) sense-position $\left(x^{\prime}, y^{\prime}\right)$ must be possible in $s^{\prime}$, which by (12) means $y^{\prime}=\operatorname{position}\left(s^{\prime}\right)$ and $y^{\prime} \in\left[x^{\prime}-c, x^{\prime}+c\right]$. Putting these together we see that position $\left(s^{\prime}\right) \in[x-c, x+c]$. Furthermore, again by (7), we obtain position $\left(s^{\prime+}\right) \in[x-c, x+c]$. Hence, the position in every $K$-related state lies in a fixed range, so the agent must know this.

There are a couple of points worth noting. First, if the agent has very incomplete knowledge of its position, then its knowledge of its position will increase after reading its sensor. This increase of knowledge arises from the pruning away of $K$-related situations that have divergent values of position. In particular, in situations with extreme values of position there will be no observationally indistinguishable action sense-position $\left(x, y^{\prime}\right)$ that can be executed (the fact that $y^{\prime}$ must equal the situation's position value will violate the bound between $y^{\prime}$ and the fixed value of $x$ ).

Second, if the agent has strong knowledge of its position, then that knowledge will not degrade after reading its sensor. For example, suppose that the agent knows the exact value of its current position. This means that position has the same value in all situations $K$-related to the current situation. In this case, the agent will continue to know its exact position after reading its sensor. This arises from the fact that all situations that are $K$ related to the successor state must arise from the execution of a sense-position action in a situation that was $K$-related to the current state. Since, sense-position actions do not change the value of position, they will all continue to share the same value of position.

\section{$5 \quad$ Likelihood and Degree of Belief}

Asserting bounds on the difference between the nominal values and the actual values in actions like sense-position $(x, y)$ represents a rather weak model of action uncertainty. Suppose, for example, we have a sensor with an error bound of $c=2$, and we make a number of readings of a particular fluent using the sensor, all of which are clustered around the value 3. For concreteness, suppose they are all between 2.8 and 3.1. As far as knowledge goes, all the agent will be able to conclude is that it knows the fluent to have a value in the range [1.1,4.8]. Getting numerous readings of 3 will not change this knowledge. Yet, even if the agent is using a cheap sensor, we might hope that getting many such readings would increase the agent's degree of belief that the true value of the fluent is indeed close to 3 .

To formalize these intuitions, we first quantify the notion of possibility (as captured by the $K$ fluent) by associating with each world the agent considers possible the agent's degree of belief that that is the actual world. We can think of this degree of belief as a subjective probability. We then consider how these degrees of belief change over time, as a result of actions being performed.

Degrees of Belief. It is convenient to capture degree of belief by first associating with each situation a weight, and then taking the degree of belief to be the normalized weight (since, like probability, we want the degree of belief in the whole space to be 1). We capture 
the weight by using a new functional fluent $p\left(s^{\prime}, s\right)$, analogous to $K\left(s^{\prime}, s\right)$. This function denotes the relative weight given to situation $s^{\prime}$ by the agent when it is in situation $s$.

We expect weights to be non-negative and that all situations considered impossible will be given weight 0 . The following constraints, which we assume is part of the background action theory ensures that this is indeed the case initially:

$$
\forall s . p\left(s, S_{0}\right) \geq 0 \wedge \neg K\left(s, S_{0}\right) \supset p\left(s, S_{0}\right)=0 .
$$

As we shall see, the successor-state axiom for $p$ ensures that these constraints hold at all times.

We take the agent's degree of belief in $\phi$ to be the total weight of all the worlds he considers possible where $\phi$ holds, normalized by the total weight of all worlds he considers possible. Thus, we are restricting to discrete probability distributions here, where the probability of a set can be computed as the sum of the probabilities of the elements of the set. We introduce special notation for this, just as we did for KNOW.

Definition 6: $[\mathrm{BEL}]$ Let $s_{\text {now }}$ be a special situation term. We write $\operatorname{BEL}\left(\phi\left[s_{\text {now }}\right], s\right)$ to denote the agent's degree of belief in $\phi\left[s_{\text {now }}\right]$ when it is in situation $s$. This is an abbreviation for the term

$$
\sum_{\left\{s^{\prime}: \phi\left[s_{\text {now }} / s^{\prime}\right]\right\}} p\left(s^{\prime}, s\right) / \sum_{s^{\prime}} p\left(s^{\prime}, s\right) .
$$

As with KNOw, we sometimes suppress the $s_{\text {now }}$ terms. In Appendix $\mathbb{A}$, we show how the summations in this formula can be expressed using second-order quantification. A logical consequence of this formalization is that $\operatorname{BEL}(\cdot, s)$ is a probability distribution over the situations $K$-related to $s$.

Action-Likelihood Functions. Next we have to define a successor-state axiom for the $p$ fluent, that is, we need to consider how the agent's probability distribution changes over time. It will not be necessary to characterize how belief changes, since belief is defined in terms of $p$.

Suppose the agent performs an action $a$ in some situation $s$. If $a$ is an ordinary deterministic action like pickup $(x)$, then the agent knows it will end up in a unique situation $d o(\operatorname{pickup}(x), s)$. Intuitively, in this case, the probability that it ascribed to $s$ will be transferred to $d o(\operatorname{pickup}(x), s)$. Similarly, this is true for all the situations that the agent considers possible at $s$.

But suppose that $a$ is an action like sense-position $(x, y)$ : sensing a value of $x$ when the true position is $y$. In this case, the probability the agent ascribes to $d o(a, s)$ will not simply be what is transferred from $s$; how likely the action $a$ is must also be taken into account. As discussed above, sense-position $(x, y)$ is not an action that an agent can execute directly: it executes something like noisy-sense-position, and one of the sense-position actions is selected nondeterministically. But some of these are more likely to be selected than others. For example, if the true position in situation $s$ is 3.0, we expect that sense-position $(3.0,3.0)$ is far

8 This is in contrast to continuous probability distributions, in which the probability of each point is 0 , so the probability of a set cannot be computed as the sum of the probabilities of the elements in the set. Instead we must integrate over the set. 
more likely to be executed than sense-position(30.0,3.0). Consequently, the probability the agent ascribes to the situation $d o$ (sense-position(3.0,3.0), $s$ ) should be greater than what it ascribes to $d o$ (sense-position $(30.0,3.0), s$ ).

To specify these probabilities, we use what we call an action-likelihood function. We add a special function $\ell(a, s)$ to our language, used to denote the probability (assigned by the agent in situation $s$ ) of primitive action $a$ being selected among all its possible observationally indistinguishable alternatives. Thus, for example, $\ell$ (sense-position $(3.1,3.0), s)$ is the likelihood that the agent would sense 3.1 when its true position was 3.0 in situation $s$. Note that if $a$ is an ordinary deterministic action like pickup $(x)$, then its only observationally indistinguishable alternative is $a$ itself, so, as long as $a$ is possible in $s$, we would normally have $\ell(a, s)=1$.

The error profile of the various effectors and sensors available to the agent is clearly application dependent, and it is this error profile that is captured by the $\ell(a, s)$ function. Hence, we assume that the user specifies a collection of axioms characterizing $\ell$ for each action $a$.

Definition 7: [Action-Likelihood Axioms] Action-likelihood axioms are axioms of the form

$$
\ell(a, s)=z \equiv \phi(a, s)
$$

Here $\phi(a, s)$ is a formula that characterizes the conditions under which action $a$ has likelihood $z$ in situation $s$. We will see some example axioms below.

With this machinery, we can give the successor-state axiom for $p$, and hence characterize how belief changes as the result of performing an action. Suppose situation $s^{\prime+}$ is of the form $d o\left(a^{\prime}, s^{\prime}\right)$, that is, it is the result of doing action $a^{\prime}$ in situation $s^{\prime}$. Then the weight that the agent assigns to $s^{\prime+}$ in $d o(a, s)$ is the product of the weight it assigns to being in situation $s^{\prime}$ and the likelihood of $a^{\prime}$ having been performed: the former is 0 unless $K\left(s^{\prime}, s\right)$ holds, in which case it is $p\left(s^{\prime}, s\right)$; the latter is 0 unless $a^{\prime}$ is observationally indistinguishable from $a$ and possible in situation $s^{\prime}$, in which case it is $\ell\left(a^{\prime}, s^{\prime}\right)$.

Definition 8: [Successor-State Axiom for $p$ ] The successor-state axiom for $p$ is

$$
\begin{aligned}
& \operatorname{Poss}(a, s) \supset p\left(s^{\prime+}, d o(a, s)\right)= \\
& \text { if } \exists a^{\prime}, s^{\prime} . s^{\prime+}=d o\left(a^{\prime}, s^{\prime}\right) \wedge \operatorname{POss}\left(a^{\prime}, s^{\prime}\right) \wedge \operatorname{OI}\left(a, a^{\prime}, s\right) \wedge K\left(s^{\prime}, s\right) \\
& \quad \text { then } p\left(s^{\prime}, s\right) \times \ell\left(a^{\prime}, s^{\prime}\right) \\
& \text { else } 0 .
\end{aligned}
$$

Note that the axiom captures the fact that any situation that is not $K$-related to the successor state will be assigned probability 0: the precondition to the then clause is precisely the condition required for a situation to be $K$ related to the successor state (Defn. (1). It also ensures that when $\operatorname{Poss}\left(a^{\prime}, s^{\prime}\right)$ is false, the weight assigned to the situation $d o\left(a^{\prime}, s^{\prime}\right)$ will be 0.9

\footnotetext{
${ }^{9}$ This can also be achieved by eliminating Poss from this axiom and requiring $\ell\left(a^{\prime}, s^{\prime}\right)$ to be 0 when $\operatorname{Poss}\left(a^{\prime}, s^{\prime}\right)$ is false.
} 
As a concrete example of how action-likelihood axioms can be used, consider an effector action like advance $(x, y)$, where the agent chooses the nominal value $x$ and the actual value $y$ is nondeterministic. Suppose the correlation between $x$ and $y$ is described by a linear Gaussian model: the actual value $y$ is the nominal value $x$ plus some random noise that has a normal distribution. This means that $y-x$, the difference between the actual movement and the nominal movement, is normally distributed, with some mean $\mu$ and variance $\sigma^{2}$. If we further assume that the effector is calibrated so that there is no systematic bias, then $\mu=0$, and the distribution of $y-x$ is characterized by $\operatorname{Normal}((y-x) / \sigma)$, where $\operatorname{Normal}(z)$ is the standardized normal distribution with mean 0 and variance 1. tro

Given these assumptions, the following axiom describes the action:

$$
\ell(\operatorname{advance}(x, y), s)=\operatorname{Normal}((y-x) / \sigma) .
$$

This axiom captures the linear Gaussian error model, asserting that the probability of the effector moving $y$ units when asked to move $x$ units falls off like the normal distribution as the difference between these two values increases. We can do something similar for sensor actions. If $x$ is the value read and $y$ is the actual value, we might once again assume that their difference $x-y$ is normally distributed, say with variance ${\sigma^{\prime 2}}^{2}$ and mean 0. (Again, assuming a mean of 0 implies that there is no systematic bias in the reading.) This gives us the following action-likelihood axiom:

$$
\ell(\text { sense-position }(x, y), s)= \begin{cases}\operatorname{Normal}\left((y-x) / \sigma^{\prime}\right) & \text { if } \operatorname{position}(s)=y \\ 0 & \text { if } \operatorname{position}(s) \neq y\end{cases}
$$

which specifies that the sensor's error profile has a normal distribution, but with perhaps a different variance $\sigma^{\prime 2}$. In either case, we could have also insisted that $\ell(a, s)$ be 0 when $\operatorname{Poss}(a, s)$ is false (for example, when the difference between the nominal and actual value exceeds some bound), but as noted above, this is not necessary because of the way we have written the successor-state axiom for $p$.

Example 5.1: When specifying the likelihood function we can encode complex contextdependent error profiles. Suppose have a fluent slippery that is true of situations where the robot is located on a slippery surface. We could account for a context-dependent effect on the robot's motion effectors with an axiom like

$$
\begin{aligned}
& \ell(\operatorname{advance}(x, y), s)= \\
& \text { if } \operatorname{slippery}(s) \\
& \text { then } \operatorname{Normal}((x-y) / 2.4) \\
& \text { else } \operatorname{Normal}((x-y) / 0.5)
\end{aligned}
$$

\footnotetext{
${ }^{10}$ There is a slight subtlety here. The normal distribution is, of course, a continuous distribution, so the probability of a single real number is always zero. Only non-trivial intervals of the real line have positive probability. We are assuming that all probability functions are discrete here, so we really need to consider an approximation to the normal distribution. In examples like this, we can do so by treating action parameters as finite precision numbers. In this case, we interpret $\operatorname{Normal}(z)$ as being the integral of the normal density function over the range of precision of $z$. For example, if the actuator allows the agent to ask for only movements like 3.1 with 2 digits of precision, the range denoted by 3.1 could be 3.05-3.15, and Normal(3.1) would be the value of the integral of the normal density function over this range. Given some fixed precision, a table of values for Normal can be computed and added to the domain theory as a set of equations. From here on in, when we write Normal, we actually mean the discrete probability distribution that approximates Normal.
} 
which indicates that the probability of error increases significantly when the surface is slippery (the standard deviation increases). To the best of our knowledge, no other formalism for representing the uncertainty associated with sensors and effectors allows this sort of context-dependent error profile to be expressed.

Similarly, suppose that the effector's error was cumulative. That is, the further the robot tried to move the greater the error. Then an action-likelihood axiom like the following could be used:

$$
\ell(\operatorname{advance}(x, y), s)=\operatorname{Normal}((x-y) /(c x))
$$

This axiom specifies that the standard deviation is some factor, $c$, of the nominal distance the agent is attempting to move.

\section{Summary of the Formalization}

This concludes the formal details of our approach. But before looking at some its properties, let us summarize the components. To incorporate noisy sensors and effectors as well as degrees of belief into the situation calculus, we start with the situation calculus language from before, but add two additional fluents, $K$ and $p$, a new distinguished predicate Or and function $\ell$. We need to extend the basic theory of action (see Section 2.3) to what we will now call an extended theory of action, consisting of the following axioms:

- axioms describing the initial situation, as before, but now including Axiom 14 for $K$ and $p$ in $S_{0}$;

- action-precondition axioms, as before;

- successor-state axioms, as before, but now including one for $K$ (Defn. đ) and one for $p$ (Defn. 8);

- unique-name axioms for the primitive actions, as before;

- observation-indistinguishability axioms (Defn. 3) characterizing Or, one for each action;

- action-likelihood axioms (Defn. 7) characterizing $\ell$, one for each action;

- various abbreviations for convenience, including KNOW, BEL, and Do;

- axioms (given in Appendix A) to ensure that BEL is always a probability distribution.

- the foundational domain-independent axioms. These need to be modified to take into account that because of its epistemic alternatives, $S_{0}$ is no longer the only initial situation 12]. However, nothing we do here hinges on these details.

As we shall show, all of the expected properties are then logical consequences of these axioms. In taking this approach, we obtain the full expressiveness of the situation calculus for reasoning about action, a simple solution to the frame problem, as well as an expressive language for dealing with the uncertainty arising from sensors and effectors. 


\section{The Formalism at Work}

We have presented a particular model for noisy actions and their effects on an agent's beliefs. Our model involves using nondeterministic actions with probabilities attached to the various nondeterministic choices. This gives a model of actions that is essentially identical to standard probabilistic actions, where an action yields various successor states with varying probabilities. Although this is a fairly standard model, it is difficult to defend its correctness in any formal way. The best that can be done is to demonstrate that the model behaves in a way that matches our intuitions and properly replicates other more specialized approaches.

\subsection{Knowledge-producing actions}

In the Scherl and Levesque approach, in addition to ordinary actions like pickup $(x)$, there are assumed to be special knowledge-producing action associated with various fluents. For example, to open a safe by dialing its combination, an agent needs to know what the combination is. If the combination of the safe is written on a piece of paper, there might be a knowledge-producing action read-combi whose effect is to change the the state of knowledge to make the value of the combi fluent known.

We can model this type of action as follows. Assume we have a primitive action read $(z)$ which is the action of reading the number $z$ on the piece of paper. We assume that this action only happens if $z$ is the combination of the safe, which we model using a precondition axiom.

$$
\operatorname{Poss}(\operatorname{read}(z), s) \equiv z=\operatorname{combi}(s)
$$

In this case, there is no distinction between the number observed on the paper and the actual value, and so this action is observationally indistinguishable only from itself:

$$
\mathrm{OI}(\operatorname{read}(z), a, s) \equiv a=\operatorname{read}(z)
$$

Finally, the agent cannot choose which number to see, it can only choose whether or not to see what is written on the paper. Consequently, we define read-combi by

$$
\text { read-combi } \stackrel{\text { def }}{=} \pi z \cdot \operatorname{read}(z) \text {. }
$$

It is then easy to show, that after reading what is on the paper, the agent knows the combination to the safe. That is, the following is entailed:

$$
D o\left(\text { read-combi, } s, s^{+}\right) \supset \exists z \cdot \operatorname{KNOW}\left(z=\operatorname{combi}, s^{+}\right) .
$$

This approach clearly generalizes to handling knowledge-producing actions which tell the agent the truth value of some fluent. One nice advantage of the approach here is that, unlike in Scherl and Levesque, it is not necessary to mention by name the knowledge-producing actions in the successor-state axiom for $K$, which means that it is not necessary to know them all in advance. 


\subsection{The FHMV Model of Knowledge}

To further understand our approach to modeling the agent's knowledge and belief in the situation calculus, it is useful to compare it with the approach to modeling multi-agent systems taken in [9, 6] (and expanded in [10] to allow probabilistic actions). In this approach, agents are always assumed to be in some local state. Besides the agents, there is an environment, which at any given time is also in a particular state. The environment's state captures everything that is relevant to the description of the system and not captured by the agent's state. Thus, if there is only one agent (as is the case here), the system's global state at any time can be described by a pair $\left(s_{e}, s_{a}\right)$, where $s_{e}$ is the environment's state and $s_{a}$ is the agent's state. The system's global state changes as a result of actions being performed. These actions may change either (or both) of the components of the global state. For example, a sensing action that did not change the environment could be modeled as changing only the agent component $s_{a}$ of the global state.

What we are doing here using the situation calculus can be modeled within this framework by mapping situations to global states. That is, we map every situation $s$ to a pair $\left(s_{e}, s_{a}\right)$. This mapping can be constructed in a variety of ways. Perhaps the easiest is to take $s_{e}$, the environment's state, to consist of the values of the fluents (other than $d o, K$, and $p$ ) in $S_{0}$, together with the sequence of primitive actions used to generate the situation. Similarly, the agent's state, $s_{a}$, can consist of an initial state that describes the agent's beliefs in $S_{0}$, together with a sequence that records what the agent learns as a result of performing the actions described in the corresponding environment state. In our approach, the agent learns only that an observationally indistinguishable action was executed; so an appropriate sequence for the agent state can be generated by replacing each action $a$ in the corresponding environment sequence by a unique canonical representative from the set of actions that are observationally indistinguishable to $a$ (with respect to the situation that $a$ was executed in). We remark that Lakemeyer independently used this approach to give semantics to knowledge and only knowing in the situation calculus [11] (also see [12]). This method of mapping situations to global states captures the implicit situation calculus assumption that the agent remembers all of the actions that have been performed (modulo observationally indistinguishability). It is also obvious that from any global state $\left(s_{e}, s_{a}\right)$ we have sufficient information to reconstruct the situation $s$ that gave rise to it.

With this view of situations, the definition of $d o$ for the FHMV model is immediate. If $\left(s_{e}, s_{a}\right)$ is the global state corresponding to situation $s$, then $d o\left(a,\left(s_{e}, s_{a}\right)\right)$ is the new global state $\left(s_{e}^{\prime}, s_{a}^{\prime}\right)$ where $s_{e}^{\prime}$ is the result of appending $a$ to $s_{e}$, and $s_{a}^{\prime}$ is the result of appending a canonical member of $\{x: \operatorname{Or}(a, x, s)\}$ to $s_{a}$. Similarly, there is an obvious definition for $K$ as well, which is the same as that taken in [9, 27]: $K\left(\left(s_{e}^{\prime}, s_{a}^{\prime}\right),\left(s_{e}, s_{a}\right)\right)$ holds precisely if $s_{a}^{\prime}=s_{a}$, i.e., the agent has the same local state. Notice that this makes $K$ an equivalence relation, that is, reflexive, symmetric, and transitive. Finally, we can model $p$ by defining $p\left(\left(s_{e}^{\prime}, s_{a}^{\prime}\right),\left(s_{e}, s_{a}\right)\right)$ to be equal to $p\left(s^{\prime}, s\right)$ where $s^{\prime}$ is the situation corresponding to the global state $\left(s_{e}^{\prime}, s_{a}^{\prime}\right)$. By normalizing summations over $p$ we obtain a distribution that defines the agent's probabilistic beliefs when it is in global state $\left(s_{e}, s_{a}\right)$.

Besides helping to clarify our approach to modeling the agent's epistemic state, the discussion above also shows that the situation calculus is not fundamental. We could have 
taken essentially the same approach using a modal logic (the FHMV model is formalized in terms of modal logic) instead.

\subsection{Bayesian conditioning}

A standard model for belief update in the light of noisy information is Bayesian conditioning. The standard Bayesian model assumes two pieces of probabilistic information: a prior distribution $\operatorname{Pr}(t)$ on the value $t$ being sensed, and a conditional distribution $\operatorname{Pr}(x \mid t)$ that gives the probability of sensing $x$ given that the true value is $t$. Furthermore, the standard model requires the assumption that the value read from the sensor is dependent only on the true value, and is thus independent of other factors given this value.

Bayes' Rule is now applied to obtain a posterior probability $\operatorname{Pr}(t \mid x)$ over the values of $t$, given that the sensor read the value $x$ : $\operatorname{Pr}(t \mid x)=\operatorname{Pr}(x \mid t) \operatorname{Pr}(t) / \operatorname{Pr}(x)$. The denominatorthe prior probability of reading the value $x$-is the only unknown expression, but it can be easily computed. Since $\sum_{t^{\prime}} \operatorname{Pr}\left(t^{\prime} \mid x\right)=1$, we must take $\operatorname{Pr}(x)$ to be the normalizing factor $\sum_{t^{\prime}} \operatorname{Pr}\left(x \mid t^{\prime}\right) \operatorname{Pr}\left(t^{\prime}\right)$. Since this normalizing factor is independent of $t$, we see that the key factor in determining the posterior probabilities is the numerator $\operatorname{Pr}(x \mid t) \operatorname{Pr}(t)$, which describes the relative probability of different values of $t$ given the observation $x$.

Two significant assumptions are typically made when applying Bayesian conditioning. The first is that the world does not change while the observation is being made, and the second is that conditioning on a value (like $x$ above) is the same as conditioning on the event of observing $x$.

In general, there need not be a distinction between sensing actions, that only affect the agent's beliefs, and ordinary actions that affect the agent's environment: in our formalism it is quite possible to define actions that change the world in the course of making an observation. For such actions, conditioning the agent's beliefs only on the value sensed (as is done above) does not adequately describe the agent's new belief state. It is necessary to also take into account the effect of the other changes caused by the action (this is the so-called "total evidence" requirement of Bayesian conditioning). In our formalism the agent's new beliefs (as described by the updated $p$ ) is in fact affected by both the sensed value and any other changes caused by an action.

To understand the second assumption, imagine that there are two fluents, Light-On and Book, where Light-On(s) holds if the light is on in situation $s$ and Book(s) holds if there is a book in the room. Initially, say that the agent considers the four possible situations characterized by these two fluents as equally likely. Suppose the agent does a sensing action that detects whether or not the book is in the room, but only if the light is on; when the light is off nothing can be determined. If the sensing action does in fact detect the book, then the agent should ascribe probability 1 to the light being on. However, this is not the probability obtained by simply conditioning the initial uniform distribution on Book. The probability that the light is on given that the book is in the room is $1 / 2$, but the probability that the light is on given that the book was observed to be in the room is 1 . The naive application of Bayes' rule implicitly assumes that conditioning on the book being in the room is equivalent to conditioning on observing the book.

In such examples, we are dealing with a sensing action that is dependent on more than just 
the value (or truth) of the fluent being sensed. It depends on other features of the situation as well. As demonstrated in Example 5.1, our formalism can model context-dependent sensing actions, and the agent's new beliefs will reflect both the values sensed as well as what can be learned about the context from that value. In this case, sensing the value true for the fluent Book also allows us to learn that Light-On is true.

Our formalism does not force us to make these two assumptions, but when we do our approach updates beliefs in a manner identical to standard Bayesian conditioning. We demonstrate this in the next section.

\subsubsection{Noisy sensors}

To see how our formalism works, suppose the agent can perform a noisy sensing action noisy-sense- $f$ to sense the functional fluent $f$. This action is the nondeterministic union of the primitive actions sense- $\mathrm{f}(x, y)$, which denotes that $x$ is sensed, but the true value of $f$ is $y$. As we would expect, the precondition for the primitive action is

$$
\text { Poss }(\text { sense-f }(x, y), s) \equiv y=f(s)
$$

the action sense- $\mathrm{f}(x, y)$ is possible in situation $s$ exactly if $f(s)=y$. We assume that the likelihood of these actions is otherwise independent of $s$, being dependent only on the actual value of $f$ (which is equal to $y$ by the above precondition) and the value sensed:

$$
\ell(\text { sense- } \mathrm{f}(x, y), s)=\ell(\text { sense- } \mathrm{f}(x, y)) .
$$

Note that this assumption is satisfied if we assume that the likelihood is a linear Gaussian function of $x-y$, as discussed in Section 5. We take noisy-sense- $\mathrm{f}(x)$ to denote the complex action of observing $x$ on the sensor; thus,

$$
\text { noisy-sense- } \mathrm{f}(x) \stackrel{\text { def }}{=} \pi y \text {. sense- } \mathrm{f}(x, y) \text {. }
$$

We now want to make sure that the assumptions underlying Bayesian updating hold. To capture the first assumption, that the world does not change while the observation is being made, we simply ensure that sense- $\mathrm{f}(x, y)$ does not affect any other fluent besides $K$ and $p$. In particular, using (4) and (5), we ensure that the action does not appear in the positive or negative effect axioms for any other fluent.

That the second assumption holds is almost immediate: the value $x$ returned by the sensing action is influenced only by the true value of $f$.

Now we derive a characterization of the agent's beliefs after reading the value $x$ from the sensor. Suppose that $s^{+}$is a possible successor state after reading the sensor, i.e., $D o$ (noisy-sense- $\left.\mathrm{f}(x), s, s^{+}\right)$holds, so that $s^{+}$is the result of executing sense- $\mathrm{f}(x, y)$ in situation $s$, for some $y$ and a fixed value of $x$. From the precondition of sense-f $(x, y)$, we see that we must have $y=f(s)$, so there is actually only one possible successor state; that is, $s^{+}=d o($ sense- $\mathrm{f}(x, f(s)), s)$. Since sense-f does not affect $f$, we also have $f\left(s^{+}\right)=f(s)$.

From $p$ 's successor-state axiom (Defn. 8), we have that $p\left(s^{+}, s^{+}\right)$can be nonzero only for situations $s^{\prime+}$ that are the result of executing an observationally indistinguishable action whose preconditions are satisfied in a state $s^{\prime}$. From the axioms for sense-f, we see that all 
such situations with non-zero weight must be of the form $s^{+}=d o$ (sense-f $\left.\left(x, f\left(s^{\prime}\right)\right), s^{\prime}\right)$, so $p\left(s^{\prime^{+}}, s^{+}\right)=p\left(s^{\prime}, s\right) \ell\left(\right.$ sense-f $\left.\left(x, f\left(s^{\prime}\right)\right), s^{\prime}\right)$ for some $s^{\prime}$, and $f\left(s^{\prime+}\right)=f\left(s^{\prime}\right)$.

Since BEL is an abbreviation for summations over $p$ (Defn. 6), we obtain

$$
\begin{aligned}
& \text { Do (noisy-sense-f } \left.(x), s, s^{+}\right) \supset \\
& \quad \operatorname{BEL}\left(f\left(s_{\text {now }}\right)=t, s^{+}\right) \\
& \quad=\sum_{\left\{s^{\prime+}: f\left(s^{\prime+}\right)=t\right\}} p\left(s^{+}, s^{+}\right) / \sum_{s^{\prime}+} p\left(s^{\prime+}, s^{+}\right) \\
& \quad=\sum_{\left\{s^{\prime}: f\left(s^{\prime}\right)=t\right\}} p\left(s^{\prime}, s\right) \ell\left(\text { sense- } \mathrm{f}\left(x, f\left(s^{\prime}\right)\right), s^{\prime}\right) / \sum_{s^{\prime}} p\left(s^{\prime}, s\right) \ell\left(\text { sense-f }\left(x, f\left(s^{\prime}\right)\right), s^{\prime}\right) .
\end{aligned}
$$

It is not hard to show that the numerator of this last expression is the (unnormalized) belief that the reading is $t$ and $x$ is observed (which is the product of the prior belief that $t$ will be read and the conditional probability of observing $x$ given $t$ ), while the denominator is the (unnormalized) belief that $x$ will be read. Thus, this computation simulates the Bayesian computation. This comes out even more clearly if we take advantage of our assumption that the likelihood is independent of $s$. With this assumption, the term $\ell$ (sense- $\left.f\left(x, f\left(s^{\prime}\right)\right), s^{\prime}\right)$ is constant over the summation in the numerator. By dividing both numerator and denominator by $\sum_{s^{\prime}} p\left(s^{\prime}, s\right)$, we can convert the sums over $p$ into an expression involving BEL:

$$
\begin{aligned}
& D o\left(\text { noisy-sense- } \mathrm{f}(x), s, s^{+}\right) \supset \\
& \qquad \operatorname{BEL}\left(f\left(s_{\text {now }}\right)=t, s^{+}\right)=\frac{\operatorname{BEL}\left(f\left(s_{\text {now }}\right)=t, s\right) \ell(\text { sense- } f(x, t))}{\sum_{t^{\prime}} \operatorname{BEL}\left(f\left(s_{\text {now }}\right)=t^{\prime}, s\right) \ell\left(\text { sense- } f\left(x, t^{\prime}\right)\right) .}
\end{aligned}
$$

So we see that the belief that the value of $f$ is $t$ after doing a sensing action is the product of the belief that the value of $f$ was $t$ before doing the sensing action and the conditional probability of reading the value $x$ given that the true value is $t$, divided by a normalizing factor. Furthermore, if we examine the denominator, we see that it can be interpreted as the agent's prior belief that it would read the value $x$ from its sensor. Again, our calculation is identical to the standard Bayesian calculation.

\subsubsection{Noisy effectors}

Noisy effectors modify the agent's beliefs in a similar manner. The key difference here is that these actions also change the world, so the agent's updated beliefs must reflect those changes.

Suppose that we have an action change- $\mathrm{f}(x, y)$ that can be used to modify the current value of the fluent $f$. Let its precondition be

$$
\operatorname{POSS}(\text { change-f }(x, y), s) \equiv \text { TRUE }
$$

(indicating that the action is always executablem), its likelihood function be

$$
\ell(\text { change- } \mathrm{f}(x, y), s)=\ell(\text { change- } \mathrm{f}(x, y))
$$

\footnotetext{
${ }^{11}$ This is similar to (9), the precondition to advance, except that we are not asserting any absolute error bound.
} 
(indicating that the likelihood is not dependent on the situation), and its corresponding nondeterministic action (denoting that the effector was actuated with the nominal value $x$ ) be

$$
\text { noisy-change- } \mathrm{f}(x) \stackrel{\text { def }}{=} \pi y \text {. change- } \mathrm{f}(x, y) \text {. }
$$

Furthermore, suppose that the only effect of change-f is to modify the fluent $f$ (and suppose that it is the only action that does so). This is reflected in $f$ 's successor-state axiom, which is

$$
\begin{gathered}
\operatorname{Poss}(a, s) \supset f(d o(a, s))= \\
\text { if } \exists x, y \cdot a=\text { change- } f(x, y) \\
\text { then } f(s)+y \\
\text { else } f(s) .
\end{gathered}
$$

Suppose that the agent attempts to increment the fluent $f$ by executing the action noisy-change-f $(x)$. From the precondition we can see that there are many different situations $s^{+}$such that $D o$ (noisy-change-f $(x), s, s^{+}$) (unlike the case above for noisy sensors, where there was a unique successor situation). Each of these situations is the result of executing change- $\mathrm{f}(x, y)$, for some $y$, in situation $s$. That change-f changes the world is captured by the fact that for each of these successor situations $s^{+}=d o($ change-f $(x, y), s)$, we have $f\left(s^{+}\right)=f(s)+y$.

As in our derivation for noisy sensors, we have

$$
\begin{aligned}
& \text { Do(noisy-change- } \left.\mathrm{f}(x), s, s^{+}\right) \supset \\
& \operatorname{BEL}\left(f\left(s_{\text {now }}\right)=t, s^{+}\right) \\
& =\sum_{\left\{s^{\prime+}: f\left(s^{\prime+}\right)=t\right\}} p\left(s^{+}, s^{+}\right) / \sum_{s^{\prime}} p\left(s^{\prime+}, s^{+}\right) \\
& =\sum_{y} \sum_{\left\{s^{\prime+}: f\left(s^{+}\right)=t \wedge s^{\prime+}=d o\left(\text { change- } \mathrm{f}(x, y), s^{\prime}\right)\right\}} p\left(s^{\prime+}, s^{+}\right) / \sum_{s^{\prime}} p\left(s^{\prime+}, s^{+}\right) \\
& =\sum_{y} \sum_{\left\{s^{\prime}: f\left(s^{\prime}\right)=t-y\right\}} p\left(s^{\prime}, s\right) \ell\left(\text { change-f }(x, y), s^{\prime}\right) / \sum_{y} \sum_{s^{\prime}} p\left(s^{\prime}, s\right) \ell\left(\text { change-f }(x, y), s^{\prime}\right) \\
& =\sum_{y} \ell(\text { change-f }(x, y)) \sum_{\left\{s^{\prime}: f\left(s^{\prime}\right)=t-y\right\}} p\left(s^{\prime}, s\right) / \sum_{y} \ell(\text { change- } f(x, y)) \sum_{s^{\prime}} p\left(s^{\prime}, s\right) \text {. }
\end{aligned}
$$

Now dividing top and bottom by $\sum_{s}^{\prime} p\left(s^{\prime}, s\right)$ to convert to beliefs, and simplifying, using the observation that $\sum_{y} \ell($ change- $\mathrm{f}(x, y))$ is 1 , we obtain

$$
\begin{aligned}
& D o\left(\text { noisy-change-f }(x), s, s^{+}\right) \supset \\
& \quad \operatorname{BEL}\left(f\left(s_{\text {now }}\right)=t, s^{+}\right)=\sum_{y} \ell(\text { change-f }(x, y)) \operatorname{BEL}\left(f\left(s_{\text {now }}\right)=t-y, s\right) .
\end{aligned}
$$

There are two differences between this result and our previous result for noisy sensors. First, we have a summation over $y$, the possible values that could have been generated by the action. This summation arises from the fact that any situation prior to the action could have given rise to a situation in which $f=t$ : all that is required is that an action with the appropriate effect on $f$ be executed. In the noisy-sensor case, the actual value of the fluent is not changed, so we need to consider only the prior situations in which the fluent has the same value. Second, it seems that there is no normalizing constant in this case. However, the normalizing constant is present and equal to $\sum_{t^{\prime}} \sum_{y} \ell($ change- $f(x, y)) \operatorname{BEL}\left(f\left(s_{\text {now }}\right)=t^{\prime}-y, s\right)$. It turns out that this sum is always equal to 1 .

There is another way of deriving this result. Consider the agent's beliefs about the value of the fluent $f$ prior to executing change-f. The fluent $f$ can be viewed as being 
a random variable, with the probability that it takes on any particular value $t$ equal to $\operatorname{BEL}\left(f\left(s_{\text {now }}\right)=t, s\right)$. When the action noisy-change- $\mathrm{f}(x)$ is executed, the value $y$ that it adds to $f$ is also a random variable with distribution $\ell($ change- $f(x, y))$. Hence, the new value of $f$ after executing noisy-change- $\mathrm{f}(x)$ is the sum of two independent random variables, the old value of $f$ and the amount added $y$. A standard result from probability theory is that the distribution of the sum of two independent random variables is the convolution of their distributions. Our result above shows that the agent's new beliefs about $f$ are in fact the convolution of its prior beliefs and the distribution on the amount by which $f$ can change.

\subsection{Examples}

It is convenient to be able to talk about what happens to the agent's beliefs and knowledge after reading some particular sequence of values from its sensors. To do this, we introduce a parameterized version of noisy-sense-position:

$$
\text { noisy-sense-position }(x) \stackrel{\text { def }}{=} \pi y \text {. sense-position }(x, y)
$$

where only the value $y$ is chosen (by the value of position in the situation). Intuitively, noisy-sense-position $(x)$ is the "action" where the agent activates its sensor and then observes that the value $x$ has been returned. This action is not actually executable by the agent, since it cannot choose to execute a noisy-sense-position(3.0) over, say, a noisy-sense-position(2.8). Nevertheless, we can write $D o$ (noisy-sense-position(3.0), $s, s^{+}$), which asserts that the agent has executed a noisy-sense-position and read the value 3.0 from its sensor, ending up in $s^{+}$.

Example 7.1: Suppose that the agent is sensing its position position using noisy-sense-position actions. For the purposes of this example, let the action-likelihood axiom for sense-position be

$$
\begin{aligned}
& \ell(\text { sense-position }(x, y), s)=\text { if } x=y \\
& \text { then } 0.5 \\
& \text { else if }|x-y|=1 \\
& \text { then } 0.25 \\
& \text { else } 0
\end{aligned}
$$

Here we are assuming that position and the arguments to sense-position can take on only integer values (i.e., this is the precision of these numbers). The axiom specifies that there is zero probability that the sensor will read a position that is greater than 1 unit away from the true position, and that the sensor noise is independent of other features of the situation.

Let the agent's initial beliefs regarding position be given by $\operatorname{BEL}\left(\operatorname{position}\left(s_{\text {now }}\right)=t, S_{0}\right)=$ $1 / 8$, for $t \in\{8,9,11,12\}$, and $\operatorname{BEL}\left(\right.$ position $\left.\left(s_{\text {now }}\right)=10, S_{0}\right)=1 / 2$. Initially, the agent does not ascribe positive probability to any other possible value for position. This distribution of beliefs for the various values of position in $S_{0}$ is shown in Figure 1 .

Suppose that the agent senses its position and observes the value 11. If $S_{1}$ is a possible successor situation, so that $D o$ (noisy-sense-position(11), $S_{0}, S_{1}$ ) holds, a simple calculation using (17) shows how the agent's beliefs change: $\operatorname{BEL}\left(\operatorname{position}\left(s_{\text {now }}\right)=10, S_{1}\right)=4 / 7$, $\operatorname{BEL}\left(\operatorname{position}\left(s_{\text {now }}\right)=11, S_{1}\right)=2 / 7$, and $\operatorname{BEL}\left(\operatorname{position}\left(s_{\text {now }}\right)=12, S_{1}\right)=1 / 7$. The new distribution is also shown in Figure 1. Since, with probability 1, the sensor returns a value 


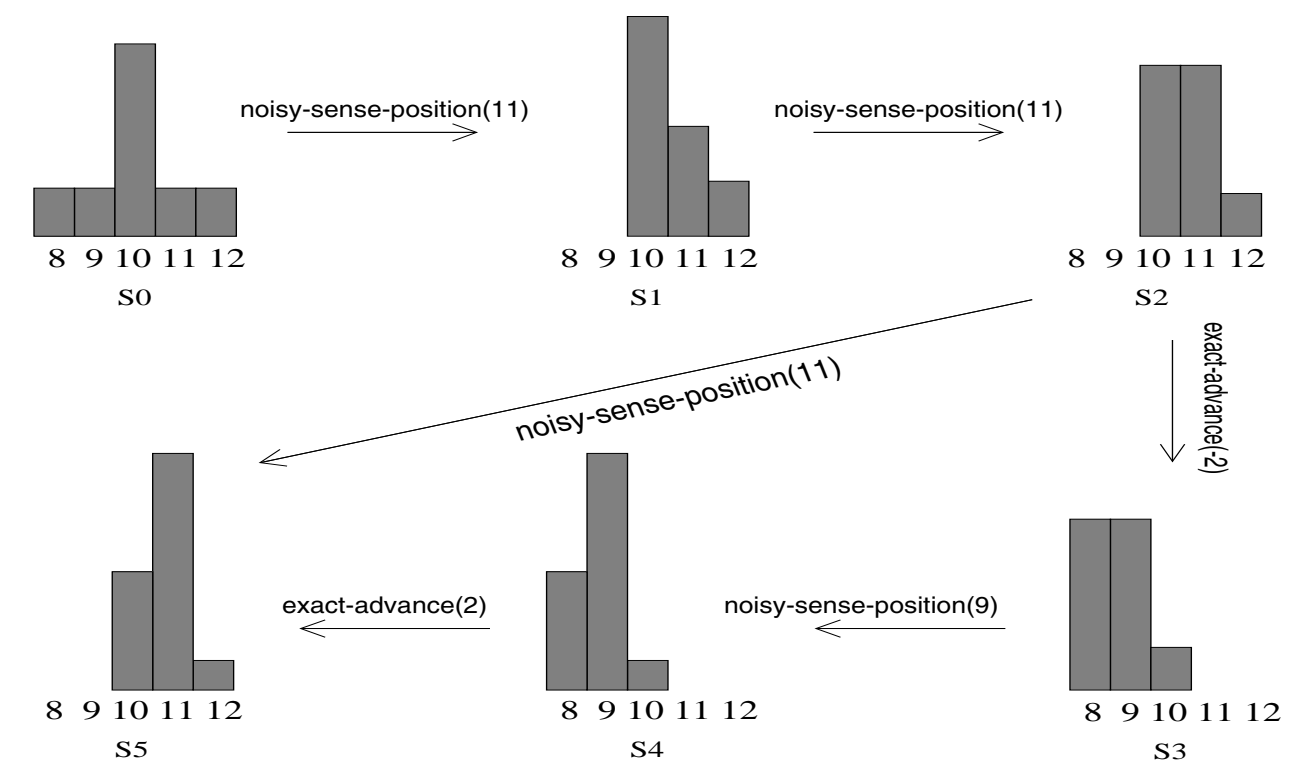

Figure 1: Sensing with Exact Motion

that is within 1 unit of the true value, the agent now has degree of belief zero in the values 8 and 9 .

Note that Figure 1 shows that the agent still that the most likely value of position is 10, even though its sensor returned the value 11. This arises from the agent's high prior belief in the value being 10 .

We have not specified an absolute error bound for sense-position, i.e., a value for $c$ in (12), implicitly assuming it to be greater than 1 . If, for example, we were to specify that the agent knows that the sensor cannot return an error of greater than 2 , i.e., if we set $c=2$ in the precondition axiom, then we will have that the agent knows that position $\neq 8$. However, the agent will not know that position $\neq 9$, even though it still has zero degree of belief in this value. Our framework distinguishes between full belief and knowledge in this case.

Sequences of sensor readings of the same fluent, including sequences of readings from different sensors, are also handled correctly in our framework. Such sequences correspond to sequences of sensing actions, and thus are handled by a simple iteration of (17), given that the sensors satisfy the assumptions of that equation. In particular, if the sensor action likelihood function is dependent only on the actual value being sensed, then each sensor reading will be independent of all previous readings. ${ }^{12}$ As a result, after a sequence of sensing actions, the agent will come to have either greater or less certainty about the value of the sensed fluent, depending on whether or not the sequence of readings agree or not.

Example 7.2 : Suppose that the agent executes another noisy-sense-position action in

\footnotetext{
${ }^{12} \mathrm{~A}$ situation is a world history consisting of the sequence of actions executed (Section 2). Hence, one can easily write a sensor-likelihood axiom that did display a dependency on previous readings, if, for example, one wanted to capture the properties of a sensor that displayed some form of hysteresis.
} 
the situation $S_{1}$. Further, suppose that the agent observes the same value as before 11, and let $S_{2}$ be such that Do(noisy-sense-position(11), $S_{1}, S_{2}$ ). Then, another application of (17) (applied to the agent's beliefs in $S_{1}$ ), yields the belief distribution shown in Figure 1: $\operatorname{BEL}\left(\right.$ position $\left.\left(s_{\text {now }}\right)=10, S_{2}\right)=4 / 9, \operatorname{BEL}\left(\right.$ position $\left.\left(s_{\text {now }}\right)=11, S_{2}\right)=4 / 9$, and $\operatorname{BEL}\left(\right.$ position $\left.\left(s_{\text {now }}\right)=12, S_{2}\right)=1 / 9$. The agent's beliefs have converged more tightly around the value 11 , since it has now sensed that value twice.

The most important part of our formalism is the natural manner in which effectors and sensors interact in their effects on the agent's beliefs. For simplicity, first we examine the case where the agent has exact control over a fluent, but can sense its value only approximately. Then we examine the case of a noisy sensor interleaved with a noisy effector.

Example 7.3: Suppose that instead of the noisy effector noisy-advance, the agent has an exact effector action exact-advance that has no preconditions, and affects position as specified in (6). Note that exact-advance is an ordinary deterministic action, so it is observationally indistinguishable only from itself, and it has a unit likelihood function: $\ell$ (exact-advance $(x), s)=1$.

Let the agent move exactly 2 units backwards when it is in situation $S_{2}$. Call the new situation $S_{3}=d o$ (exact-advance $\left.(-2), S_{2}\right)$. Then, the successor-state axiom for $p$ and position imply that the agent's beliefs are precisely shifted to worlds in which it has moved backward 2 units: we have a simple transfer of probability mass from each situation to its successor situation in which the agent has moved backward 2 units.

Deterministic actions like this modify the agent's beliefs in a manner that is related to Lewis's notion of imaging [16]. In imaging, beliefs are updated by transferring all probability mass to the "closest" world, rather than by renormalizing the mass after removing some worlds as when we condition. Here, every situation transfers its probability mass to its successor. Different things are true in these successor situations, as the action has effected various changes. This means that the agent will believe different things in the successor situation. Nevertheless, since all of these successor situations arise from the execution of the same action, the changes to the agent's beliefs are generally systematic. In particular, in the absence of ramifications, it will change its beliefs only about fluents affected by the action.

In this example, we have that $\operatorname{BEL}\left(\operatorname{position}\left(s_{\text {now }}\right)=t, S_{3}\right)=\operatorname{BEL}\left(\operatorname{position}\left(s_{\text {now }}\right)=\right.$ $\left.t+2, S_{2}\right)$ for all $t$. The agent's shifted beliefs are shown in Figure 1. This is exactly how one would expect the agent's beliefs to change in response to an exact movement like this.

Example 7.4: Suppose that the agent again executes a noisy-sense-position action in $S_{3}$ and observes the value 9 . Let $S_{4}$ be such that $D o$ (noisy-sense-position $(9), S_{3}, S_{4}$ ). A reading of 9 is consistent with the previous readings of 11 , since the agent has moved back 2 units. Hence, as shown in Figure 1, it results in a further tightening of the agent's beliefs, around the value 9. If the agent subsequently moves forward 2 units, executing an exact-advance(2) action, so that $S_{5}=d o$ (exact-advance $\left.(2), S_{4}\right)$, its beliefs will then be clustered around 11 , as shown in the figure: $\left.\operatorname{BEL}\left(\operatorname{position}\left(s_{\text {now }}\right)=10, S_{5}\right)=4 / 13, \operatorname{BEL}\left(\operatorname{position}\left(s_{\text {now }}\right)=11, S_{5}\right)=8 / 13\right)$, and $\operatorname{BEL}\left(\right.$ position $\left.\left(s_{\text {now }}\right)=12, S_{5}\right)=1 / 13$.

Intuitively, since the agent's exact-advance action incurs no error, we would expect that if the agent had sensed the value 11 in situation $S_{2}$, then its beliefs about the distance to 
the wall should not change after moving forwards and backwards an equal distance. Our model respects this intuition. In particular, the agent's beliefs in $S_{5}$ are identical to what they would be if it had executed an noisy-sense-position(11) in $S_{2}$, as indicated in the figure by the diagonal arrow from $S_{2}$ to $S_{5}$.

Example 7.5: Now we examine the case of a noisy effector. Let us return to the effector noisy-advance, taking (77) to specify the successor-state axioms for position. Suppose that the likelihood axiom for advance is

$$
\begin{aligned}
& \ell(\operatorname{advance}(x, y), s)= \text { if } x=y \\
& \text { then } 0.5 \\
& \text { else-if }|x-y|=1 \\
& \text { then } 0.25 \\
& \text { else } 0
\end{aligned}
$$

Here, with probability 1, the difference between the agent's actual move and the move specified is no more than one unit.

Starting at situation $S_{5}$, with beliefs about position as given above, suppose that $S_{6}$ is a successor state of an attempt to move forward 1 unit, so that $D o$ (noisy-advance $(1), S_{5}, S_{6}$ ) holds. The likelihood axiom above indicates that in this case the probability the agent moves forward 2 units is $1 / 4,1$ unit is $1 / 2$, and 0 units is $1 / 4$. Equation 18 can be used to calculate the agent's new beliefs about its position. In particular, we obtain $\operatorname{BEL}\left(\operatorname{position}\left(s_{\text {now }}\right)=\right.$ $\left.10, S_{6}\right)=4 / 52, \operatorname{BEL}\left(\right.$ position $\left.\left(s_{\text {now }}\right)=11, S_{6}\right)=16 / 52, \operatorname{BEL}\left(\operatorname{position}\left(s_{\text {now }}\right)=12, S_{6}\right)=$ $21 / 52, \operatorname{BEL}\left(\right.$ position $\left.\left(s_{\text {now }}\right)=13, S_{6}\right)=10 / 52$, and $\operatorname{BEL}\left(\operatorname{position}\left(s_{\text {now }}\right)=14, S_{6}\right)=1 / 52$. This distribution is shown in Figure 2.

Suppose that the agent now attempts to move back to its previous position by executing a noisy-advance $(-1)$. If situation $S_{7}$ is such that $D o$ (noisy-advance $\left.(-1), S_{6}, S_{7}\right)$ holds, then the agent's beliefs in $S_{7}$ are $\operatorname{BEL}\left(\operatorname{position}\left(s_{\text {now }}\right)=8, S_{7}\right)=1 / 52, \operatorname{BEL}\left(\operatorname{position}\left(s_{\text {now }}\right)=\right.$ $\left.9, S_{7}\right)=6 / 52, \operatorname{BEL}\left(\operatorname{position}\left(s_{\text {now }}\right)=10, S_{7}\right)=14.25 / 52, \operatorname{BEL}\left(\operatorname{position}\left(s_{\text {now }}\right)=11, S_{7}\right)=$ $17 / 52, \operatorname{BEL}\left(\right.$ position $\left.\left(s_{\text {now }}\right)=12, S_{7}\right)=10.5 / 52, \operatorname{BEL}\left(\operatorname{position}\left(s_{\text {now }}\right)=13, S_{7}\right)=3 / 52$, and $\operatorname{BEL}\left(\right.$ position $\left.\left(s_{\text {now }}\right)=14, S_{7}\right)=0.25 / 52$.

We see from this example that in the presence of noisy motion, the agent's confidence in its location degrades; its beliefs spread out. And this effect increases the more it moves. Unless it wants to lose its way completely, it must resort to sensing. Figure 2 shows what happens to the agent's beliefs after it senses its position to be 11 in situation $S_{7}$. If $S_{8}$ is such that $D o$ (noisy-sense-position(11), $\left.S_{7}, S_{8}\right)$ holds, then the agent's new beliefs in $S_{8}$ are $\operatorname{BEL}\left(\operatorname{position}\left(s_{\text {now }}\right)=8, S_{8}\right)=0, \operatorname{BEL}\left(\operatorname{position}\left(s_{\text {now }}\right)=9, S_{8}\right)=0, \operatorname{BEL}\left(\operatorname{position}\left(s_{\text {now }}\right)=\right.$ $\left.10, S_{8}\right)=57 / 235, \operatorname{BEL}\left(\right.$ position $\left.\left(s_{\text {now }}\right)=11, S_{8}\right)=136 / 235, \operatorname{BEL}\left(\operatorname{position}\left(s_{\text {now }}\right)=12, S_{8}\right)=$ $42 / 235, \operatorname{BEL}\left(\right.$ position $\left.\left(s_{\text {now }}\right)=13, S_{8}\right)=0$, and $\operatorname{BEL}\left(\right.$ position $\left.\left(s_{\text {now }}\right)=14, S_{8}\right)=0$. Thus, the beliefs are considerably sharpened by the sensing operation.

The previous examples have employed discrete beliefs and likelihood functions, which make the computations more apparent. More practical models are also easily accommodated, and in fact sometimes yield simpler, albeit less obvious, computations. 


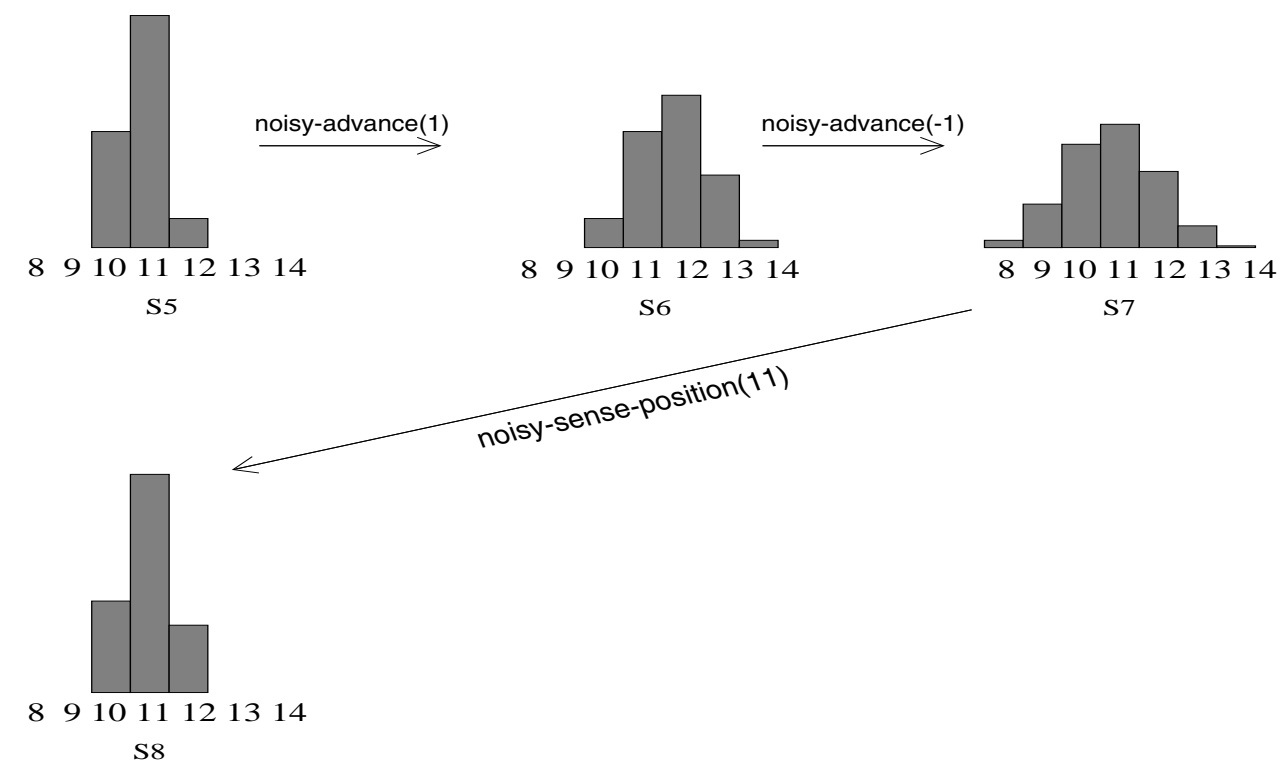

Figure 2: Noisy Motion

In robotics applications one of the most popular models of noisy sensors and effectors is the linear Gaussian model where the nominal value is the actual value plus a Gaussian noise factor (see, e.g., the various papers in [4]). Equations 15 and 16 are examples of linear Gaussian models. The next example shows how easy belief update is with such models.

Example 7.6: Suppose that the agent has an action noisy-change-f for effecting changes to a fluent $f$, and an action noisy-sense-f for sensing its value, as in Sections 7.3.1 and 7.3.2. Further, suppose that the likelihood functions for both change-f and sense-f are linear Gaussian, so that

$$
\ell(\text { change-f }(x, y), s)=\operatorname{Normal}\left((y-x) / \sigma_{e}\right),
$$

and

$$
\ell(\text { sense- } \mathrm{f}(x, y), s)=\operatorname{Normal}\left((y-x) / \sigma_{s}\right) .
$$

Suppose that the agent's initial beliefs about a fluent $f$ are described by the normal distribution $N\left(\mu_{0}, \sigma_{0}^{2}\right)$ with mean $\mu$ and variance $\sigma_{0}^{2}$. That is, $\operatorname{BEL}\left(f\left(s_{\text {now }}\right)=t, S_{0}\right)=$ $\operatorname{Normal}\left(\left(t-\mu_{0}\right) / \sigma_{0}\right)$, where $\operatorname{Normal}(z)$ is the standardized normal distribution with mean 0 and variance 1 .

Now suppose that a noisy-change- $\mathrm{f}\left(x_{1}\right)$ action is executed. Equation 18 shows that the agent's new beliefs about $f$ is that its value is the result of adding two independent random variables (which gives rise to the convolution of two distributions). In particular, the new value for the fluent $f$ is the sum of the change actually generated by the action and $f$ 's previous value. The likelihood axiom for change- $f\left(x_{1}, y\right)$ indicates that $y$, the change to $f$ generated by the action, is a normally distributed random variable with mean $x_{1}$ and variance $\sigma_{e}^{2}$. The agent's initial beliefs indicate that $f$ 's previous value is also a normally distributed random variable with mean $\mu_{0}$ and variance $\sigma_{0}^{2}$. 
A well known (and easy-to-derive) result is that the sum of two normally distributed random variables is also normally distributed (e.g., see [18, page 146]). In particular, we have that, after executing the action, the agent's new beliefs about $f$ are described by another normal distribution with mean $\mu_{1}=\mu_{0}+x_{1}$ and variance $\sigma_{1}^{2}=\sigma_{0}^{2}+\sigma_{e}^{2}$.

Next, suppose that the agent executes a sensing action and reads the value $z_{1}$ from its sensor, i.e., the action noisy-sense- $\mathrm{f}\left(z_{1}\right)$ is executed. Equation 17 indicates that the agent's new beliefs about the value of $f$ are the normalized result of multiplying its prior beliefs by the probability of observing $z_{1}$ given the value of $f$. Both of these quantities are again described by normal distributions. The prior beliefs are normally distributed, and for each value of $f$, the probability of $z_{1}$ is also normally distributed, according to the likelihood function above. In this case, another well-known result is that the posterior beliefs about $f$ remain normally distributed (see, e.g., [19, page 2]). In particular, we have that the agent's new beliefs about $f$ are that its value is normally distributed with mean $\mu_{2}=\left(z_{1} \sigma_{1}^{2}+\mu_{1} \sigma_{s}^{2}\right) /\left(\sigma_{s}^{2}+\sigma_{1}^{s}\right)$ and variance $\sigma_{2}^{2}=\sigma_{1}^{2} \sigma_{s}^{2} /\left(\sigma_{1}^{2}+\sigma_{s}^{2}\right)$.

It is easy to see that these equations can be applied iteratively to keep track of the agent's beliefs about a fluent's value given a sequence of modification to and sensing of this value. With a normal distribution we need keep track only of the current mean and variance to completely describe the agent's beliefs.

The fact that a normal distribution is preserved under these types of updates, with easy-to-compute modifications to its parameters, forms the basis of the popular technique of Kalman filtering [3]. In fact, this example is simply an instance of Kalman filtering, and it can be generalized within our framework to handle the situation where the fluent being modified and sensed is vector valued. In this case the above variances would be replaced by covariance matrices, and the computations to update the mean and variance would become matrix manipulations.

All of the previous examples involved the sensing or affecting of a numeric fluent. Our final example demonstrates that the formalism can also be used to model noisy actions that affect non-numeric fluents.

Example 7.7: In Section 1 a we used the example of dropping fragile objects. However, when a fragile object is dropped it does not always break, it breaks with some probability. We an model this situation quite easily in our formalism.

Suppose that there are two primitive drop actions, drop-break and drop-not-break, characterized by the following axioms:

$$
\begin{gathered}
\text { Poss }(\operatorname{drop}-\operatorname{break}(x), s) \equiv \operatorname{Holding}(x, s) \wedge \operatorname{Fragile}(x, s) \\
\operatorname{Poss}(\operatorname{drop}-\operatorname{not}-\operatorname{break}(x), s) \equiv \operatorname{Holding}(x, s) \\
\text { OI }\left(\operatorname{drop}-\operatorname{break}(x), a^{\prime}, s\right) \equiv a^{\prime}=\operatorname{drop-break}(x) \vee a^{\prime}=\operatorname{drop}-\operatorname{not}-\operatorname{break}(x) \\
\text { OI }\left(\operatorname{drop}-\operatorname{not}-\operatorname{break}(x), a^{\prime}, s\right) \equiv a^{\prime}=\operatorname{drop-not-break}(x) \vee a^{\prime}=\operatorname{drop}-\operatorname{break}(x) \\
\ell(\operatorname{drop}-\operatorname{break}(x), s)=\text { if } \operatorname{Fragile}(x) \quad \text { then } 0.8 \quad \text { else } 0 \\
\ell(\operatorname{drop}-\operatorname{not}-\operatorname{break}(x), s)=\text { if } \operatorname{Fragile}(x) \quad \text { then } 0.2 \quad \text { else } 1 .
\end{gathered}
$$


Let the successor-state axiom for Broken be

$$
\operatorname{Poss}(a, s) \supset \operatorname{Broken}(x, d o(a, s)) \equiv a=\operatorname{drop}-\operatorname{break}(x) \vee \operatorname{Broken}(x, s) .
$$

Finally, suppose that the agent can execute a nondeterministic action drop:

$$
\operatorname{drop}(x) \stackrel{\text { def }}{=} \operatorname{drop}-\operatorname{break}(x) \mid \operatorname{drop}-\operatorname{not}-\operatorname{break}(x) .
$$

These axioms specify that the actions drop-break and drop-not-break cannot be distinguished by the agent. That is, the agent can execute a drop action, but does not know ahead of time whether or not dropping an object will cause it to be broken. If the object is not fragile only the drop-not-break action can be executed, in which case it has probability 1 of being executed. On the other hand, if the object is fragile, then the preconditions of both drop-not-break and drop-break are satisfied and either can be executed. The probability of drop-break being executed, i.e., the probability that a fragile object will break when dropped, is 0.8 , while drop-not-break has probability 0.2 . The successor-state axiom for break specifies that drop-break does in fact cause an object to become broken.

If the agent executes $\operatorname{drop}(x)$ in situation $s$ for some object $x$ given that KNOW(holding $(x), s)$ (so that the action is possible), then we obtain for any successor situation $s^{+}$

$$
\begin{aligned}
& \operatorname{Do}\left(\operatorname{drop}(A), s, s^{+}\right) \wedge \operatorname{KNOw}(h o l d i n g(x), s) \\
& \quad \supset \operatorname{BEL}\left(\operatorname{Broken}\left(x, s_{\text {now }}\right), s^{+}\right)=0.8 \times \operatorname{BEL}\left(\operatorname{Fragile}\left(x, s_{\text {now }}\right), s\right) .
\end{aligned}
$$

For example, if the agent's degree of belief is 0.5 that the object being dropped is fragile, then the agent will have degree of belief degree 0.4 that the object will be broken after being dropped. Similarly, if the agent is certain that the object is fragile, then it will have degree of belief 0.8 that the object will break after being dropped. This makes sense intuitively, as the object can be broken only by dropping if it was originally fragile, and even then there is a probability of only 0.8 that it will be broken by the drop action.

\section{Conclusion}

We have demonstrated that noisy perception and actions can be modeled in the situation calculus by a simple extension of previous work. In particular, from the successor-state axiom for $p$, (8), and a constraint on its values in $S_{0}$, (14), we obtain as consequences what many have argued to be the natural models for belief update from perception (Bayesian conditioning) and from actions (a form of Lewis's imaging) [23]. Most importantly, our formalism succeeds in capturing some key features of the interaction between these two models for belief change.

Much of our approach can be exported to alternate formalisms. For example, instead of the situation calculus a modal logic could have been used. Similarly, the probabilistic component could be replaced with an alternate formalism, like Dempster-Shafer belief functions [30] or possibility measures [5]. All that would be required is to replace the functional fluent $p$ and axioms for BEL with fluents and axioms to support an alternate measure of belief. The likelihood functions could then be replaced with non-probabilistic functions to support an alternate rule of belief update. 
As for future work, apart from addressing limitations of the formalism, there is its application to high-level agent control. In the GOLOG work mentioned in the introduction, the ability of an agent to execute a program depends on what it knows about the truth value of the test conditions in that program [13]. When an agent has only a degree of belief in the truth of a test condition in a program it can randomize the program's execution. That is, instead of executing a set of actions only when a test condition is true, it can randomly decide to execute the actions with probability $p$ when its degree of belief in the test condition is $p$. The key issue will then be characterizing the program's effects. For example, one could construct programs that with probability bounded below by $1-\epsilon$ achieve a certain condition. Or one could employ a richer model that included an assignment of utilities to situations, and attempt to construct programs that maximize the agent's expected utility.

\section{A Formalization of Belief}

In this appendix, we show how BEL can be formalized so as to ensure that it is in fact a probability distribution.

As mentioned in Section 2, we treat the situation calculus as a many-sorted dialect of the predicate calculus with some second-order features. Included among the sorts are situations and real numbers. For convenience, we also assume that the language includes the natural numbers as a sub-sort of the reals. [1] In particular, in the language we have variables that range only over these specific sorts.

With sorted variables we can then formalize the summations used in our definition of BEL (Defn. 6). Let $\phi\left(s_{\text {now }}\right)$ be a formula over the special situation term $s_{\text {now }}, r$ and $r^{\prime}$ be variables of sort real, $f$ and $g$ be second-order function variables (ranging over all functions), and $m, i$, and $j$ be variables of sort natural number. We define $\operatorname{sum}_{p}\left(\phi\left(s_{\text {now }}\right), s\right)$ to be the summation of $p$ in $s$ over all situations $s^{\prime}$ satisfying $\phi\left(s_{\text {now }} / s^{\prime}\right)$ :

$$
\begin{aligned}
& \operatorname{sum}_{p}\left(\phi\left(s_{\text {now }}\right), s\right)=r \stackrel{\text { def }}{=} \\
& \forall r^{\prime} .\left(r^{\prime}<r\right) \equiv \\
& \exists f, g, m . \\
& \forall i, j .(i \neq j \supset g(i) \neq g(j)) \wedge\left(i \leq m \supset \phi\left(s_{\text {now }} / g(i)\right)\right) \\
& \wedge f(0)=0 \\
& \wedge \forall i . f(i+1)=f(i)+p(g(i), s) \\
& \wedge f(m)>r^{\prime}
\end{aligned}
$$

Basically, this formula says that $\operatorname{sum}_{p}\left(\phi\left(s_{\text {now }}\right), s\right)$ is equal to $r$ iff for every value $r^{\prime}, r^{\prime}$ is less than $r$ iff there exists a finite set $m$ of situations (enumerated by the function $g(0), \ldots$, $g(m)$ ) satisfying $\phi$ whose $p$ values sum to a value (computed by the function $f$ ) greater than $r^{\prime}$. Note that this definition entails that an infinite set of situations has a $p$ sum that is the

\footnotetext{
${ }^{13}$ As is well known, with 0,1 , and + in the language, we can actually define the natural numbers, since we have second-order quantification, as follows: $n \in N$ is an abbrevation for $\forall P((0 \in P \wedge \forall y(y \in P \supset y+1 \in$ $P)) \supset n \in P$ ).

${ }^{14}$ Note that we can easily do without such variables by including some unary "type" predicates in our language.
} 
limit of the sum of $p$ over its elements. In particular, the resulting probability distribution (achieved once we normalize the sum) will be a discrete distribution.

Now we can formally define $\operatorname{BEL}\left(\phi\left(s_{\text {now }}\right), s\right)$ as

$$
\operatorname{BEL}\left(\phi\left(s_{\text {now }}\right), s\right)=\operatorname{sum}_{p}\left(\phi\left(s_{\text {now }}\right), s\right) / \operatorname{sum}_{p}(\text { TRUE, } s),
$$

where TRUE is satisfied by all situations.

In Section 5 we imposed the following constraints on $p$ :

$$
\forall s .\left(p\left(s, S_{0}\right) \geq 0\right) \wedge\left(\neg K\left(s, S_{0}\right) \supset p\left(s, S_{0}\right)=0\right) .
$$

Now we can add the additional constraint:

$$
\exists r . r>0 \wedge \operatorname{sum}_{p}\left(\mathrm{TRUE}, S_{0}\right)=r,
$$

where $r$ is a variable of sort real. This constraint ensures that the sum of $p$ over all situations is, in $S_{0}$, a finite positive number. From these constraints it follows, that for every formula $\phi, \operatorname{sum}_{p}\left(\phi\left(s_{\text {now }}\right), S_{0}\right)$ exists and, since $p$ is non-negative, is less than (or equal to) $\operatorname{sum}_{p}\left(\phi\left(s_{\text {now }}\right), S_{0}\right)$. It is then immediate that in $S_{0}$, BEL is a probability distribution over the space of situations. Finally, the constraint $\neg K\left(s, S_{0}\right) \supset p\left(s, S_{0}\right)=0$ entails that all that is known is assigned degree of belief 1 .

What about BEL in situations other than $S_{0}$ ? By identical reasoning, in any situation $s$, BEL will be a probability distribution over the set of situations if the sum of $p$ over all situations is, in $s$, a finite positive number and $p$ is non-negative. To guarantee this we require that the action-likelihood functions (Defn. 7) be probability distributions over the set of actions.

First we define $\operatorname{sum}_{\ell}\left(\phi\left(a_{\text {now }}, s\right), s\right)$ to be the sum of $\ell\left(a_{\text {now }}, s\right)$ over all actions $a_{\text {now }}$ that satisfy the formula $\phi\left(a_{\text {now }}, s\right)$ in situation $s$. This can be defined in an identical manner to $\operatorname{sum}_{p}$, the only change required is to sum the values of $\ell(g(i), s)$ instead of the values of $p(g(i), s)$ in the formula above. We then impose the following constraints:

1. $\forall a, s \cdot \ell(a, s) \geq 0$.

2. $\forall a, s \cdot \operatorname{sum}_{\ell}\left(\mathrm{OI}\left(a, a_{\text {now }}, s\right), s\right)=1$.

The first formula says the action-likelihood function is non-negative, while the second formula ensures that for every action $a$ the sum of the likelihood function over all actions $a_{\text {now }}$ that are observationally indistinguishable to $a$ in situation $s$ is one. Together these two formulas make the action-likelihood function a discrete probability distribution over each set of observationally indistinguishable actions.

With this in hand, the successor-state axiom for $p$ (Defn. 8) gives us what we require. Given that $\operatorname{sum}_{p}($ TRUE, $s)$ is finite the axiom shows that for any action $a, \operatorname{sum}_{p}($ TRUE, $d o(a, s))$ remains finite since in the term $p\left(s^{\prime}, s\right) \times \ell\left(a^{\prime}, s^{\prime}\right), \ell\left(a^{\prime}, s^{\prime}\right)$ has a finite sum over the $a^{\prime}$. Since $\operatorname{sum}_{p}\left(\right.$ TRUE, $\left.S_{0}\right)$ is finite, we obtain by induction that it remains finite for every situation (since all situations arise from applying a finite sequence of actions to $S_{0}$ ). Finally, the successor-state axiom for $K$ (Defn. đ) shows that only $K$-related situations get positive values of $p$. Hence in every situation, not just $S_{0}$, all that is known is assigned degree of belief 1. 


\section{References}

[1] F. Bacchus. Representing and Reasoning with Probabilistic Knowledge. MIT Press, Cambridge, Mass., 1990.

[2] A. Darwiche and M. Goldszmidt. Action networks: A framework for reasoning about actions and change under uncertainty. In Uncertainty in Artificial Intelligence, Proceedings of Annual Conference, pages 136-144, 1994.

[3] T. L. Dean and M. P. Wellman. Planning and Control. Morgan Kaufmann, San Mateo, California, 1991.

[4] L. Dorst, M. van Lambalgen, and F. Voorbraak, editors. Reasoning with Uncertainty in Robotics. Proceedings of the International Workshop, volume 1093 of Lecture Notes in Artificial Intelligence, Amsterdam, The Netherlands, 1995. Springer.

[5] D. Dubois and H. Prade. Representation and combination of uncertainty with belief functions and possibility measures. Computational Intelligence, 4:244-264, 1988.

[6] R. Fagin, J. Y. Halpern, Y. Moses, and M. Y. Vardi. Reasoning about Knowledge. MIT Press, Cambridge, Mass., 1995.

[7] A. R. Haas. The case for domain-specific frame axioms. In F. M. Brown, editor, The Frame Problem in Artificial Intelligence: Proceedings of the 1987 workshop, pages 343348. Morgan Kaufmann, San Mateo, California, 1987.

[8] J. Y. Halpern. An analysis of first-order logics of probability. Artificial Intelligence, 46:311-350, 1990.

[9] J. Y. Halpern and R. Fagin. Modelling knowledge and action in distributed systems. Distributed Computing, 3(4):159-179, 1989. A preliminary version appeared in Proc. 4th ACM Symposium on Principles of Distributed Computing, 1985, with the title "A formal model of knowledge, action, and communication in distributed systems: preliminary report".

[10] J. Y. Halpern and M. R. Tuttle. Knowledge, probability, and adversaries. Journal of the ACM, 40(4):917-962, 1993.

[11] G. Lakemeyer. Only knowing in the situation calculus. In L. Aiello, J. Doyle, and S. Shapiro, editors, Proceedings of the International Conference on Principles of Knowledge Representation and Reasoning, pages 14-25. Morgan Kaufmann, San Mateo, California, 1996.

[12] G. Lakemeyer and Levesque H. AOL: a logic of acting, sensing, knowing and only knowing. In Proceedings of the International Conference on Principles of Knowledge Representation and Reasoning. 1998. To appear. 
[13] Y.

Lespérance, H. J. Levesque, F. Lin, and R. B. Scherl. Ability and knowing how in the situation calculus. Available via the URL ftp://ftp.cs.toronto.edu/pub/cogrob/abilityInSC.ps.Z, 1995.

[14] Y. Lespérance, H. J. Levesque, and S. Ruman. An experiment in using golog to build a personal banking assistant. In L. Cavedon, A. Rao, and W. Wobcke, editors, Intelligent Agent Systems: Theoretical and Practical Issues, volume 1209 of LNAI. Springer-Verlag, 1997.

[15] H. J. Levesque, R. Reiter, Y. Lespérance, F. Lin, and R. Scherl. Golog: A logic programming language for dynamic domains. Journal of Logic Programming, 1996. To appear in the special issue on reasoning about action and change.

[16] D. Lewis. Probability of conditionals and conditional probabilities. Philosophical Review, 83(5):297-315, 1976.

[17] F. Lin and R. Reiter. State constraints revisited. Journal of Logic and Computation, 4(5):655-678, 1994.

[18] D. V. Lindley. Introduction to Probability and Statistics. Part 1. Probablity. Cambridge University Press, Cambridge, U.K., 1965.

[19] D. V. Lindley. Introduction to Probability and Statistics. Part 2. Inference. Cambridge University Press, Cambridge, U.K., 1965.

[20] J. McCarthy and P. J. Hayes. Some philosophical problems from the standpoint of artificial intelligence. In Machine Intelligence 4, pages 463-502. Edinburgh University Press, 1969.

[21] R. C. Moore. A formal theory of knowledge and action. In J. R. Hobbs and R. C. Moore, editors, Formal theories of the common sense world, pages 319-358. Ablex Publishing, Norwood, NJ, 1985.

[22] J. Pearl. Probabilistic Reasoning in Intelligent Systems. Morgan Kaufmann, San Mateo, California, 1988.

[23] J. Pearl. Action as local surgery. In Working Notes of AAAI Spring Symposium Series, Extending Theories of Actions, pages 157-162. AAAI, 1995.

[24] E. P. D. Pednault. ADL: Exploring the middle ground between Strips and the situation calculus. In R. J. Brachman, H. J. Levesque, and R. Reiter, editors, Proceedings of the International Conference on Principles of Knowledge Representation and Reasoning, pages 324-332, 1989.

[25] J. A. Pinto. Temporal Reasoning in the Situation Calculus. PhD thesis, Department of Computer Science, University of Toronto, 1994. 
[26] R. Reiter. The frame problem in the situation calculus: A simple solution (sometimes) and a completeness result for goal regression. In V. Lifschitz, editor, Artificial Intelligence and Mathematical Theory of Computation, pages 359-380. Academic Press, New York, 1991.

[27] S. J. Rosenschein and L. P. Kaelbling. The synthesis of digital machines with provable epistemic properties. In J. Y. Halpern, editor, Theoretical Aspects of Reasoning about Knowledge: Proc. 1986 Conference, pages 83-97. Morgan Kaufmann, San Mateo, California, 1986.

[28] R. B. Scherl and H. J. Levesque. The frame problem and knowledge-producing actions. In Proceedings of the AAAI National Conference, pages 689-695, 1993.

[29] L. K. Schubert. A monotonic solution to the frame problem in the situation calculus: An efficient method for worlds with fully specified actions. In H. E. Kyburg, R. P. Loui, and G. N. Carlson, editors, Knowledge Representation and Defeasible Reasoning, pages 23-67. Kluwer Academic Press, London, 1990.

[30] G. Shafer. A Mathematical Theory of Evidence. Princeton University Press, Princeton, NJ, 1976.

[31] K. Tam, J. Lloyd, Y. Lespérance, H. Levesque, F. Lin, D. Marcu, R. Reiter, and M. Jenkin. Controlling autonomous robots with Golog. In Proc. AI-97, Perth, Australia. December 1997. 